\title{
A generic and provably convergent Shape-From-Shading Method for Orthographic and Pinhole Cameras.
}

\author{
EMMANUEL PRADOS AND OLIVIER FAUGERAS \\ Odyssée Lab., INRIA, 2004 route des Lucioles, BP 93, 06902 Sophia-Antipolis Cedex, France \\ http://www-sop/odyssee/team
}

April 27, 2005

\begin{abstract}
We describe a mathematical and algorithmic study of the Lambertian "Shape-From-Shading" problem for orthographic and pinhole cameras. Our approach is based upon the notion of viscosity solutions of Hamilton-Jacobi equations. This approach provides a mathematical framework in which we can show that the problem is well-posed (we prove the existence of a solution and we characterize all the solutions). Our contribution is threefold. First, we model the camera both as orthographic and as perspective (pinhole), whereas most authors assume an orthographic projection (see [25] for a survey of the SFS problem up to 1989 and [64, 39, 18] for more recent ones); thus we extend the applicability of shape from shading methods to more realistic acquisition models. In particular it extends the work of $[53,55]$. We provide some novel mathematical formulations of this problem yielding new partial differential equations. Results about the existence and uniqueness of their solutions are also obtained. Second, by introducing a "generic" Hamiltonian, we define a general framework allowing to deal with both models (orthographic and perspective), thereby simplifying the formalization of the problem. Thanks to this unification, each algorithm we propose can compute numerical solutions corresponding to all the modeling. Third, our work allows us to come up with two new generic algorithms for computing numerical approximations of the "continuous" solution of the "Shape-From-Shading" problem as well as a proof of their convergence toward that solution. Moreover, our two generic algorithms are able to deal with discontinuous images as well as images containing black shadows.
\end{abstract}

Keywords: Shape from Shading, Lambertian reflectance, pinhole camera, orthographic and perspective projection, black shadows, discontinuous images, viscosity solutions.

\section{Introduction}

Shape From Shading (SFS) has been a central problem in the field of computer vision since the early days. The problem is to compute the three-dimensional shape of a surface from the brightness variations in a black and white image of that surface. The work in our field was pioneered by Horn who was the first to pose the problem as that of finding the solution of a nonlinear first-order partial differential equation (PDE) called the brightness equation [26]. Later on, various approaches have been proposed: the book [25] contains a very nice survey of the research in SFS up to 1989; for a more recent overview, see $[64,39]$. Despite the richness of the literature in this area, all approaches are based on very restrictive assumptions. For example, most SFS algorithms have been developed under the assumption of orthographic projection. Few SFS approaches consider the perspective projection problem ( i.e consider a pinhole camera model instead of a simple affine model). Penna $[49,48]$ proposes a local method using geometrical properties. His formulation of the problem leads him to solve a system of algebric equations. Weiss [62] proposes a physical formalism which can exploit invariants of the imaging process and geometric knowledge about the surface. Penna [49, 48] and Weiss [62] do not present numerical results; they only de- 
scribe a theoretical method. Lee and Kuo [40] present a variational approach. They minimize a cost functional based on a local linear approximation of the reflectance map. Hasegawa and Tozzi [24] suggest to combine SFS with photogrammetry to reconstruct the surface and calibrate the camera. Their method consists in solving large systems of linear equations and seems to be suitable only for small images. More recently, Samaras and Metaras [56] propose a solution of the "perspective SFS" by using a deformable model, Yuen et al. [63] and Tankus et al. $[60,61]$ propose an adaptation of the fast marching algorithm of Kimmel and Sethian [36], Okatani and Deguchi $[43,44]$, propose an extension of the methods of propagation of the equal-height contours of Bruckstein and Kimmel [5, 34], and Tankus and Sochen [59] or Courteille et al. [11] propose some "local" methods. Let us note that in the articles [48, 49, 62, 40, 24, 56, 63] the authors do not really formulate a PDE adapted to the perspective modeling. Here, we also propose a solution of the "perspective SFS" problem, but in contrast with the previous work of $[48,49,62,40,24,56,63,59,11]$, our formalism is completely based on PDEs. Also, we formulate precise and explicit PDEs (equations $(8)^{1}$ and (10)) corresponding to the perspective SFS problem ${ }^{2}$. Note that the formulation of these new PDEs allows to prove existence and uniqueness results for the perspective SFS problem. Regarding this point, let us emphasize the importance of the questions of the existence and uniqueness of a solution of the SFS problem. These questions as well as those related to the convergence of numerical schemes for computing the solutions became central in the last decade of the 20th century. For example, the papers of Bruss [6], Brooks [4], Horn [27], and Durou [19, 20], show the difficulty of these questions. The first results related to the convergence of the numerical approximations have been presented by Dupuis and Oliensis [17] and P.-L. Lions, Rouy and Tourin [55, 42]. More recent results can be found in $[22,23,53]$. Let us mention here that all the previous theoretical work only dealt with the simplest version of the SFS problem (with orthographic projection). In this article, we deal with the same questions in the framework of the "perspective SFS" problem. Let us also re-

\footnotetext{
${ }^{1}$ Yet formulated by Prados and Faugeras in $[50,52]$ and by Tankus et al. in [59].

${ }^{2}$ Okatani and Deguchi $[43,44]$ does not make explicit the static SFS PDE. They transform the brightness equation into an evolutive equation.
}

mark that the papers of Penna [49, 48], Weiss [62], Lee and Kuo [40], Hasegawa and Tozzi [24], Samaras and Metaxas [56], Yuen et al. [63], Tankus and Sochen [59], and Courteille et al. [11] do not deal at all with these questions.

The perspective projection hypothesis extends the applicability of SFS methods to more realistic images: we can recover the shapes of objects which are located near the camera. The modeling we propose in $[50,52]$ (as that of $[48,49,40,56,63,59,11])$ assumes that the scene is illuminated by a single point light source located at infinity. In this article, we also deal with scenes which are illuminated by a single point light source located at the optical center (case also considered by Okatani and Deguchi [43, 44]). We formulate a new PDE (equation (10)), design an original algorithm and prove existence and uniqueness of a solution, thereby completing our previous work [52]. This modeling (perspective camera and light source is located at the optical center) realistically describes a simple camera equiped with a flash, or such medical imaging systems as endoscopy.

We also unify the classical model which assumes that the camera performs an orthographic projection, and the perspective model which assumes that the camera is a pinhole. To this end, we introduce a "generic" equation (equation (24)). Note that the classical SFS equations and the two new perspective SFS equations (8) and (10) are particular cases of the "generic" equation $(24)^{3}$. This generic formulation considerably simplifies the formalization of the problem. It also naturally suggests "generic" algorithms, each of which can compute numerical solutions of various perspective and orthographic SFS problems.

Finally, the algorithms we propose can deal with images containing discontinuities and black shadows. We prove the stability of our SFS approximation schemes and the convergence of our SFS algorithms when applied to such images. We also prove that our algorithms are robust to pixel noise and to the errors on the parameters of the models, e.g. the light source direction.

\footnotetext{
${ }^{3}$ Note: instead of considering equations, it would be more correct to consider "Hamiltonians"; see section 3.2.1.
} 


\section{Mathematical formulations of the Lambertian SFS problem}

The SFS problem is to recover the three-dimensional shape of a scene from the brightness variations in a black and white image of that scene.

The scene is represented by a surface $\mathbf{S}$. Let $\Omega$ be an open set of $\mathbb{R}^{2}$ representing the domain of definition of the image; for example, $\Omega$ is the rectangular domain ] $0, X[\times] 0, Y[$. We assume that $\mathbf{S}$ can be explicitly parameterized by a function $S$ from the closure $\bar{\Omega}$ of the set $\Omega$ into $\mathbb{R}^{3}$ by $x \mapsto S(x)$;

$$
\mathbf{S}=\{S(x) ; \quad x \in \bar{\Omega}\} .
$$

The image intensity is modelled as a function $I$ from $\bar{\Omega}$ into the closed interval $[0,1]$, by

$$
I: \bar{\Omega} \longrightarrow[0,1]: x \mapsto I(x) .
$$

For all $x \in \bar{\Omega}$, the intensity $I(x)$ is the brightness obtained when imaging the point $S(x)$ of the surface $\mathbf{S}$. We assume that a single point light source illuminates the scene. Thus with each point $X$ in $\mathbb{R}^{3}$ we associate the unit "light vector" $\mathbf{L}(X)$ pointing to the light source. Finally, we assume that the scene is Lambertian. We suppose that the albedo is constant and equal to 1 . For all $x$ in $\bar{\Omega}$, let us denote $\mathbf{n}(x)$, a normal vector of the surface $\mathbf{S}$ at the point $S(x)$ such that

$$
\mathbf{n}(x) \cdot \mathbf{L}(S(x)) \geq 0 .
$$

With all the above hypotheses, the brightness $I(x)$ of the point $S(x)$ of the surface $\mathbf{S}$ is the cosine of the angle $(\mathbf{n}(x), \mathbf{L}(S(x)))$. In other words:

$$
I(x)=\frac{\mathbf{n}(x) \cdot \mathbf{L}(S(x))}{|\mathbf{n}(x)|} .
$$

Note that, through differential calculus ${ }^{4}$, we can easily obtain an explicit expression for $\mathbf{n}(x)$.

\subsection{The "orthographic SFS" problem}

In this subsection we revisit one of the simplest versions of the shape from shading problem. We assume that the

\footnotetext{
${ }^{4}$ The two columns of the Jacobian $D S(x)$ are tangent vectors to $\mathbf{S}$ at the point $S(x)$. Their cross product is a normal vector.
}

light source is located at infinity. Thus, all light vectors are parallel and we can represent the light direction by a constant vector $\mathbf{L}=(\alpha, \beta, \gamma)$. We assume that the light source is above the surface, then $\gamma>0$. We note $\mathbf{l}=(\alpha, \beta)$. We assume that the camera performs an orthographic projection of the scene. With this hypothesis, it is natural to define the surface $\mathbf{S}$ by

$$
\mathbf{S}=\left\{\left(x_{1}, x_{2}, u\left(x_{1}, x_{2}\right)\right) ; \quad\left(x_{1}, x_{2}\right) \in \bar{\Omega}\right\} .
$$

So, if the plane $\left(0, \overrightarrow{x_{1}}, \overrightarrow{x_{2}}\right)$ represents the retinal plane then $|u(x)|$ is the distance of the points $S(x)$ in the scene to the camera (see figure 1). For such a surface $\mathbf{S}$, a normal vector $\mathbf{n}(x)$ is given by

$$
\mathbf{n}(x)=(-\nabla u(x), 1) .
$$

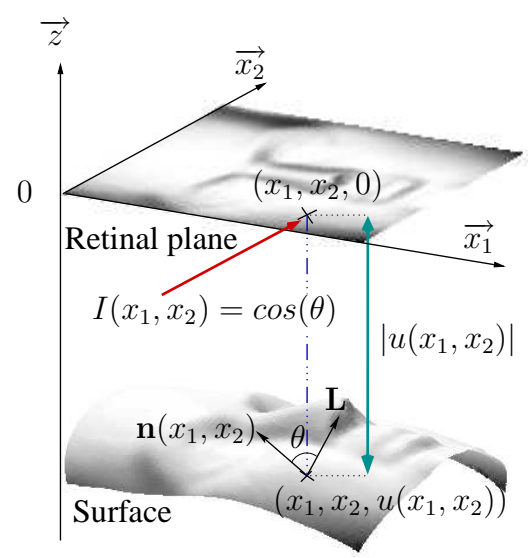

Figure 1: Image arising from an orthogonal projection. The intensity of the "pixel" $\left(x_{1}, x_{2}\right)$ is the intensity of the point $\left(x_{1}, x_{2}, u\left(x_{1}, x_{2}\right)\right)$ on the surface $\mathbf{S}$; (we assume that the camera and the light source are above the surface).

Given these hypotheses, the brightness equation (1) becomes

$$
\forall x \in \Omega, \quad I(x)=\frac{-\nabla u(x) \cdot \mathbf{l}+\gamma}{\sqrt{1+|\nabla u(x)|^{2}}}
$$

and therefore the shape from shading problem is, given an image $I$ and a light source direction $\mathbf{L}$, find a function 
$u: \bar{\Omega} \longrightarrow \mathbb{R}$ satisfying the equation:

$$
\forall x \in \Omega, \quad I(x) \sqrt{1+|\nabla u(x)|^{2}}+\nabla u(x) \cdot \mathbf{l}-\gamma=0 .
$$

Note that by using the change of variables

$$
v(x)=\mathbf{l} \cdot x+\gamma u(x),
$$

(proposed by Dupuis and Oliensis [17]), the PDE (3) can be rewriten as

$\forall x \in \Omega, \quad I(x) \sqrt{|\nabla v(x)-\mathbf{l}|^{2}+\gamma^{2}}+\nabla v(x) \cdot \mathbf{l}-1=0$.

Also, in the case where the light source is in the same direction as the direction of projection (it is the case considered by Rouy and Tourin in [55]), we have $\mathbf{L}=(0,0,1)$, and the PDE (2) can be rewriten as an Eikonal equation:

$$
\forall x \in \Omega, \quad|\nabla u(x)|-\sqrt{\frac{1}{I(x)^{2}}-1}=0 .
$$

\subsection{The "perspective SFS" problem}

In this section, we assume that the camera performs a perspective projection of the scene and that the light source is located at infinity. A "pinhole" camera is represented by its retinal plane and its optical center. It is characterized by its focal length $f$; see figure 2 . We assume that the scene can be represented by a surface $\mathbf{S}$ defined by

$$
\mathbf{S}=\left\{u\left(x_{1}, x_{2}\right)\left(x_{1}, x_{2},-f\right) ; \quad\left(x_{1}, x_{2}\right) \in \bar{\Omega}\right\} .
$$

A normal vector of such a surface is given by:

$$
\mathbf{n}(x)=\left(\begin{array}{c}
f \nabla u(x) \\
u(x)+x \cdot \nabla u(x)
\end{array}\right) .
$$

As in section 2.1, we represent the light by a constant unit vector $\mathbf{L}=(\alpha, \beta, \gamma)$, with $\gamma>0$ (we suppose that the light source is above the surface $\mathbf{S})$. We note $\mathbf{l}=(\alpha, \beta)$. In this context, the irradiance equation becomes:

$$
I(x)=\frac{f \quad l \cdot \nabla u(x)+\gamma(x \cdot \nabla u(x)+u(x))}{\sqrt{f^{2}|\nabla u(x)|^{2}+(x \cdot \nabla u(x)+u(x))^{2}}} .
$$

Now, let us suppose that the points of the surface $\mathbf{S}$ are visible (according to figure 2); So $u$ verifies $\forall x \in$
$\bar{\Omega}, \quad u(x)>0$. Since equation (7) is homogeneous in $\nabla u(x)$ and $u(x)$, we can simplify it by the change of variables $^{5} v=\ln (u)$. Thus the "perspective SFS" problem consists in solving the PDE:

$I(x) \sqrt{f^{2}|\nabla v|^{2}+(x \cdot \nabla v+1)^{2}}-(f \mathbf{l}+\gamma x) \cdot \nabla v-\gamma=0$

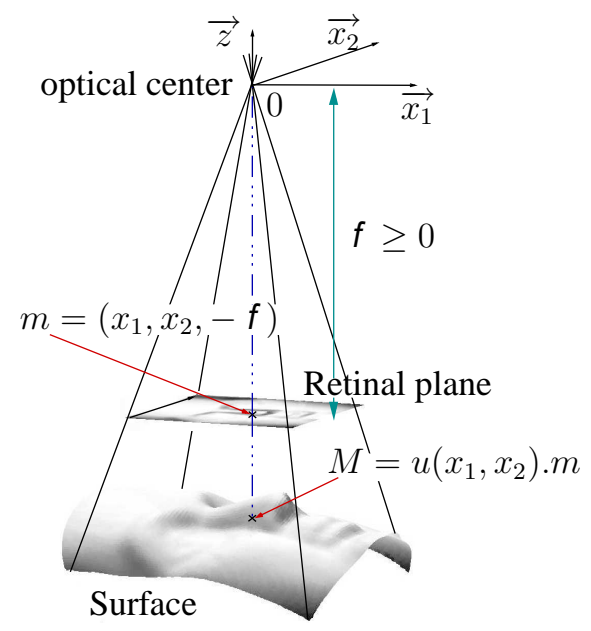

Figure 2: Image arising from a perspective projection. The intensity of the "pixel" $\left(x_{1}, x_{2}\right)$ is the intensity of the point $u\left(x_{1}, x_{2}\right)\left(x_{1}, x_{2},-f\right)$ on the surface $\mathbf{S}$; (we assume that the camera and the light source are above the surface).

\subsection{The "perspective SFS" with a point light source located at the optical center}

In this section, we assume that the camera performs a perspective projection of the scene and that the scene is illuminated by a single point light source located at the optical center. This modeling corresponds approximately to the real situation encountered when we use a camera equiped with a flash in a dark place. It also corresponds nicely to the situation encountered in some medical protocols like endoscopy in which the (point) light source is

\footnotetext{
${ }^{5}$ Also used in $[50,52,59]$.
} 


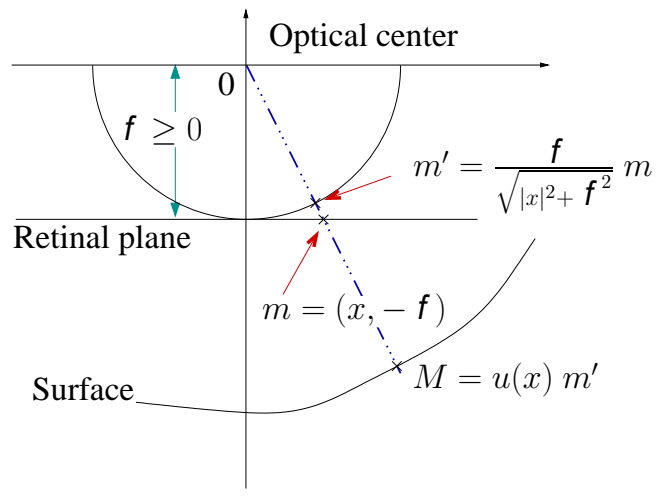

Figure 3: The intensity of the "pixel" $(x,-f)$ is the intensity of the point $u(x)(x,-f) \frac{f}{\sqrt{|x|^{2}+f^{2}}}$ on the surface $\mathbf{S}$.

located very close to the camera, because of space constraints [44]. As in section 2.2, $f \geq 0$ represents the focal length. For mathematical convenience, we change slightly the parameterization of the scene. According to figure 3, we suppose that it is represented by a surface $\mathbf{S}$ defined by

$$
\mathbf{S}=\left\{\frac{f u(x)}{\sqrt{|x|^{2}+f^{2}}}\left(\begin{array}{c}
x \\
-f
\end{array}\right) ; \quad x \in \bar{\Omega}\right\} .
$$

For such a surface $\mathbf{S}$, a normal vector $\mathbf{n}(x)$ at the point $S(x)$ is given by:

$$
\mathbf{n}(x)=\left(\begin{array}{c}
f \nabla u-\frac{f u(x)}{|x|^{2}+f^{2}} x \\
\nabla u \cdot x+\frac{f u(x)}{|x|^{2}+f^{2}} f
\end{array}\right) .
$$

The single point light source is located at the optical center, so the unit light vector $\mathbf{L}$ at point $S(x)$ is the vector

$$
\mathbf{L}(S(x))=\frac{1}{\sqrt{|x|^{2}+f^{2}}}\left(\begin{array}{c}
-x \\
f
\end{array}\right) .
$$

The irradiance equation (1) then becomes:

$$
I(x) \sqrt{\frac{f^{2}|\nabla u(x)|^{2}+(\nabla u(x) \cdot x)^{2}}{Q(x)^{2}}+u(x)^{2}}-u(x)=0 .
$$

where $Q(x)=\frac{f}{\sqrt{|x|^{2}+f^{2}}}$. Now, as in section 2.2, we suppose that the surface $\mathbf{S}$ is visible (according to figure 3 ). So $u$ verifies $\forall x \in \bar{\Omega}, \quad u(x) \geq 0$. Therefore, equation (9) being homogeneous, we can rewrite it by using the change of variables $v=\ln (u)$ :

$$
I(x) \sqrt{f^{2}|\nabla v(x)|^{2}+(\nabla v(x) \cdot x)^{2}+Q(x)^{2}}-Q(x)=0 .
$$

Note: Okatani and Deguchi [44] do not make explicit the PDE arising from the brightness equation. They design their numerical algorithm by transforming the static SFS equation as an evolution equation.

\section{Shape from Shading and viscosity solutions}

\subsection{Why using viscosity solutions to solve SFS}

The SFS PDEs (3), (6), (8) and (10) do not depend ${ }^{6}$ on $u$; so they are ill-posed. In particular, the solution is not unique. In effect, if $u$ is a solution, then for all $c \in \mathbb{R}$, $u+c$ is also a solution. The ambiguities encountered with this kind of equations are nevertheless not reduced to the translations. For example, for the eikonal equation, the concave/convexe ambiguity has been considerably studied in the SFS literature [45, 26, 33, 38, 37]. To characterize a solution, we need to impose some constraints. Let us impose Dirichlet boundary conditions (DBC) for insuring uniqueness:

$$
\forall x \in \partial \Omega, \quad u(x)=\varphi(x)
$$

$\varphi$ being a continuous real function defined on $\partial \Omega$. In other words, from the SFS point of view, we assume that the "distance" from the camera to the scene is known on the boundary of the image. Admitedly, this hypothesis may appear restrictive. In a forthcoming paper, we show how to remove these constraints. Let us note that, the reader can yet find in the SFS literature some PDEs methods computing numerical solutions with only at part of these boundary data; see for example work of Kimmel

\footnotetext{
${ }^{6}$ In the sequel, concerning equations (8) and (10), we misuse the notation of $u$, writing $u$ instead of $v$.
} 
and Bruckstein [34] and the work of Oliensis and Dupuis $[16,17,46]^{7}$.

The SFS equations (3), (5), (6), (8), and (10) are Hamilton-Jacobi equations. Generally, Hamilton-Jacobi equations with DBC do not have classical, i.e. differentiable, solutions. For example, the equation

$$
|\nabla u(x)|=1 \text { for all } x \text { in }] 0,1[
$$

with $u(0)=u(1)=0$, does not have classical solutions (Rolles theorem). The notion of viscosity solutions is a very nice way of making quantitative and operational the intuitive idea of weak solutions of first-order (and for that matter, second-order) PDEs. Also, equation (12) with the DBC $u(0)=u(1)=0$, has a (unique) continuous viscosity solution (see figure 4-a)). The notion of viscos-

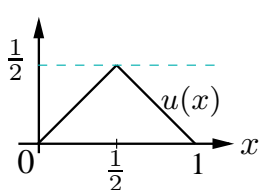

a)

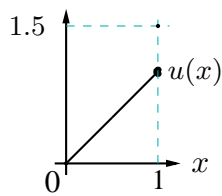

b)
Figure 4: a) Continuous viscosity solution of (12) with $u(0)=u(1)=0$; b) discontinuous viscosity solution of (12) with $u(0)=0$ and $u(1)=1.5$.

ity solutions has been introduced by Crandall and Lions $[12,41,14,13]$ in the 80 s. Its theory is now mature (see the book of Barles [2] and that of Bardi and CapuzzoDolcetta [1]) and the numerical analysis of HamiltonJacobi equations has progressed considerably (see [21]). In the shape from shading area, the first interest of the notion of viscosity solutions of Hamilton-Jacobi equations is theoretical: it allows to characterize the solutions of the SFS problem, and makes the problem well-posed. But let us emphasize that this is not the only application. In effect, Barles and Souganidis [3] have proved that the numerical solutions obtained by using monotone schemes are generally approximations of the viscosity solutions. Thus, thanks to the notion of the viscosity solutions, we can understand exactly the numerical properties of the SFS algorithms.

\footnotetext{
${ }^{7}$ It's worth to remark that all SFS methods which require not any boundary data need strong regularity properties on the solutions. In particular, in $[34,16,46]$, the global methods work only if the solution is in $C^{2}(\Omega)$.
}

In the following, we recall the definitions of viscosity solutions of Hamilton-Jacobi equations and some fundamental theorems. More details about these definitions and all proofs can be found in Barles's, Bardi and Capuzzo Dolcetta's or Lions's books [2, 1, 41].

\subsection{Viscosity solutions of Hamilton-Jacobi equations}

We start with the notion of continuous viscosity solutions introduced by Crandall and Lions [12, 41, 14].

\subsubsection{Continuous viscosity solutions}

We consider a Hamilton-Jacobi equation of the form:

$$
H(x, \nabla \mathbf{u}(x))=0, x \in \Omega,
$$

where $\Omega$ is an open subset of $\mathbb{R}^{2}$ and $H$ is a continuous real function defined by

$$
\begin{aligned}
H: \Omega \times \mathbb{R}^{2} & \longrightarrow \mathbb{R} \\
(x, p) & \longmapsto
\end{aligned}
$$

$H$ is called the Hamiltonian. The variable associated to $\nabla \mathbf{u}(x)$ is often noted $p$. Let $B U C(\Omega)$ be the set of bounded and uniformly continuous functions on $\Omega$.

\section{Definition 1 (Continuous viscosity solution)}

$u \in \operatorname{BUC}(\Omega)$ is a viscosity subsolution (respectively, a viscosity supersolution) of equation (13) if: $\forall \phi \in C^{1}(\Omega), \forall x_{0} \in \Omega$ local maximum of $(u-\phi)$,

$$
H\left(x_{0}, \nabla \phi\left(x_{0}\right)\right) \leq 0
$$

(respectively, if:

$\forall \phi \in C^{1}(\Omega), \forall x_{0} \in \Omega$ local minimum of $(u-\phi)$,

$$
\left.H\left(x_{0}, \nabla \phi\left(x_{0}\right)\right) \geq 0 \quad\right) .
$$

$u$ is a continuous viscosity solution of equation (13) if it is both a subsolution and a supersolution of (13).

Viscosity solutions are weak solutions. They are not differentiable! Nevertheless, this notion is consistent with the notion of classical solutions, as shown by the next 
Theorem 1 Let $u$ be differentiable in $\Omega$, a classical solution of (13). If $u \in B U C(\Omega)$, then $u$ is a continuous viscosity solution. Let $u$ be a continuous viscosity solution of equation (13). If $u$ is differentiable in $\Omega$, then $u$ is a classical solution.

We specify for the inexperienced reader that the definition of the viscosity solutions is associated to the Hamiltonian and not to the equation. For example, it is well known that the viscosity solutions of the Hamiltonian $H(x, p)$ are different from the viscosity solutions of the Hamiltonian $-H(x, p)$; see [51] for an example.

One of the most important interests of the viscosity solutions theory is that it provides a set of general existence and uniqueness theorems which only require very weak hypotheses. Let us recall that the SFS Hamiltonians do not depend on $u$. Thus, to have uniqueness we add boundary conditions. Our choice turns to Dirichlet conditions. Thus for the SFS problems we consider equations

$$
\left\{\begin{array}{r}
H(x, \nabla u(x))=0 \text { on } \Omega, \\
u=\varphi \text { on } \partial \Omega,
\end{array}\right.
$$

where $\varphi$ is a real function defined on $\partial \Omega$ and $H$ the adequate Hamiltonian. The following theorem 2 applies in the special case where the Hamiltonian $H$ appearing in equation (14) (hence with Dirichlet boundary conditions) is convex with respect to $\nabla u$. It ensures the existence of continuous viscosity solutions of the PDE (14). We note $H^{*}$ the Legendre transform ${ }^{8}$ of $H$ :

$$
H^{*}(x, q)=\sup _{p \in \mathbb{R}^{2}}\{p . q-H(x, p)\} \leq+\infty .
$$

Let us define $\forall x, y \in \bar{\Omega}$,

$$
L(x, y)=\inf _{\xi \in C_{x, y}, T_{0}>0}\left\{\int_{0}^{T_{0}} H^{*}\left(\xi(s),-\xi^{\prime}(s)\right) d s\right\}
$$

where $C_{x, y}$ is the set of $\xi:\left[0, T_{0}\right] \rightarrow \mathbb{R}^{2}$ such that $\xi(0)=x, \xi\left(T_{0}\right)=y, \forall t \in\left[0, T_{0}\right], \xi(t) \in \bar{\Omega}$ and $\xi^{\prime} \in L^{\infty}\left(0, T_{0}\right)$ (We denote $L^{\infty}\left(0, T_{0}\right)$ the set of bounded measurable functions defined on the interval $\left(0, T_{0}\right)$ and taking their values in $\mathbb{R}^{2}$.)

\section{Theorem 2 (Existence of continuous solutions) If}

\footnotetext{
${ }^{8}$ See appendix A of [51].
}

(H1) [convexity] $H$ is convex with respect to $p(\forall x \in \bar{\Omega})$

(H2) [uniform coercivity] $H(x, p) \rightarrow+\infty$ when $|p| \rightarrow$ $+\infty$ uniformly with respect to $x \in \bar{\Omega}$,

(H3) [subsolution] $\inf _{p \in \mathbb{R}^{2}} H(x, p) \leq 0$ in $\bar{\Omega}$,

(H4) [regularity] $H \in C\left(\bar{\Omega} \times \mathbb{R}^{2}\right)$,

(H5) [compatibility] $\forall x, y \in \partial \Omega, \varphi(x)-\varphi(y) \leq$ $L(x, y)$;

then the function $u$ defined in $\bar{\Omega}$ by:

$$
u(x)=\inf \left\{\int_{0}^{T_{0}} H^{*}\left(\xi(s),-\xi^{\prime}(s)\right) d s+\varphi\left(\xi\left(T_{0}\right)\right)\right\}
$$

is a continuous viscosity solution of equation (14) (in particular $u$ verifies $u(x)=\varphi(x)$ for all $x$ in $\partial \Omega)$.

Theorem 2 is a special case of theorem 5.3 in [41]. It can be interpreted as giving compatibility constraints for the boundary conditions. Under hypotheses (H1)-(H4), the hypothesis (H5) is a necessary and sufficient condition for the existence of the continuous viscosity solution. We will say that $\varphi$ verifies the compatibility condition if (H5) is verified.

Theorem 2 allows to prove the existence of continuous viscosity solutions of the SFS problems (see section 3.4). Nevertheless, let us point out that the existence of such a solution requires a constraint on the variation of $\varphi$ (the compatibility condition). Let us remember that in practice we can only have at best an approximation of $\varphi$. So, if we make a large error on the function $\varphi$ when we compute a numerical solution of the SFS problems and if this error is too large then there do not exist continuous viscosity solutions. For example, equation (12) with $u(0)=0, u(1)=1.5$ does not have continuous viscosity solutions, because the compatibility condition does not hold, see figure 4-b and [51]. So what do the numerical algorithms compute? In other words, how do we interpret the numerical results? It appears that as soon as there do not exist continuous viscosity solutions, we need to introduce a weaker notion of solution. It turns out that the idea of discontinuous viscosity solutions provides an answer to these problems. For instance, we can prove that equation (12) with $u(0)=0, u(1)=1.5$ has a discontinuous viscosity solution (unique in $] 0,1[$ ) which is shown in figure 
4-b). The notion of discontinuous viscosity solutions is due mostly to Ishii $[30,29]$ and is covered in detail in the book of Barles [2]. The recent book of Bardi and Capuzzo Dolcetta [1] synthesizes some recent results.

\subsubsection{Discontinuous viscosity solutions}

Let us consider the following equation on the closed subset $\bar{\Omega}$ :

$$
F(x, u(x), \nabla u(x))=0, \text { for } x \in \bar{\Omega},
$$

where $F$, defined on $\bar{\Omega} \times \mathbb{R} \times \mathbb{R}^{2}$, is the locally bounded function:

$$
F(x, u, p)= \begin{cases}H(x, p) & \text { for } x \text { in } \Omega, \\ u-\varphi(x) & \text { for } x \text { in } \partial \Omega,\end{cases}
$$

where $H$ is a real continuous function on $\bar{\Omega} \times \mathbb{R}^{2}$ and $\varphi$ is a real continuous function on $\partial \Omega$.

Definition 2 Let $u$ be a locally bounded function on a set E. $\forall x \in E$, let us note:

$$
u^{*}(x)=\limsup _{y \rightarrow x} u(y) \quad \text { and } \quad u_{*}(x)=\liminf _{y \rightarrow x} u(y)
$$

We recall also that $u: E \rightarrow \mathbb{R}$ is upper (respectively, lower) semicontinuous (u.s.c, resp. 1.s.c) if for any $x \in E$ and $\varepsilon>0$ there exists a $\delta$ such that for all $y \in E \cap B(x, \delta)$ $u(y)<u(x)+\varepsilon$ (respectively, $u(y)>u(x)-\varepsilon$ ). Note that if $u$ is a locally bounded function, then $u^{*}$ is u.s.c and $u_{*}$ is l.s.c. To familiarize oneself with these notions, the reader can refer to the sections V-1 and V-2.1 of [1].

Definition 3 (Discontinuous viscosity solutions) $A$ locally bounded function $u$, u.s.c (respectively, l.s.c) on $\bar{\Omega}$, is a discontinuous viscosity subsolution (respectively, supersolution) of equation (16) if:

$\forall \phi \in C^{1}(\bar{\Omega}), \forall x_{0} \in \bar{\Omega}$ local maximum of $(u-\phi)$,

$$
F_{*}(x, u(x), \nabla \phi(x)) \leq 0
$$

(respectively, if:

$\forall \phi \in C^{1}(\bar{\Omega}), \forall x_{0} \in \bar{\Omega}$ local minimum of $(u-\phi)$,

$$
\left.F^{*}(x, u(x), \nabla \phi(x)) \geq 0 \quad\right) .
$$

A locally bounded function $u$ is a discontinuous viscosity solution of (16) if $u^{*}$ is a subsolution and $u_{*}$ is a supersolution of (16).
Note that

$$
\begin{aligned}
& F_{*}(x, u(x), \nabla \phi(x))=F^{*}(x, u(x), \nabla \phi(x)) \\
& \quad=H(x, \nabla \phi(x)) \quad \text { if } x \in \Omega, \\
& \quad F_{*}(x, u(x), \nabla \phi(x)) \\
& \quad=\min \{H(x, \nabla \phi(x)), u(x)-\varphi(x)\} \quad \text { if } x \in \partial \Omega, \\
& \quad \begin{array}{l}
F^{*}(x, u(x), \nabla \phi(x)) \\
\quad=\max \{H(x, \nabla \phi(x)), u(x)-\varphi(x)\} \quad \text { if } x \in \partial \Omega .
\end{array}
\end{aligned}
$$

Here the idea is to include the boundary conditions in the "viscosity inequalities". Thus, we impose the boundary conditions in a weak sense. In particular, at point $x \in \partial \Omega$ where the solutions cannot be equal to $\varphi(x)$, we instead impose that the "viscosity inequalities" still hold for $H$. Let us note that the notion of discontinuous viscosity solutions extends the notion of continuous viscosity solutions. In other words, a continuous viscosity solution is a discontinuous viscosity solution. Moreover, note that a discontinuous viscosity solution can have discontinuities. For more details, we advise the reader to read chapter 4 of Barles's book [2].

The following existence theorem can be found in Bardi and Capuzzo Dolcetta's book [1] (theorem V.4.13).

Theorem 3 Let $H(x, p)=\sup _{a \in A}\{-f(x, a) \cdot p-$ $l(x, a)\}$ verifying the hypotheses (H6)-(H8) (described below). Let $\varphi \in B C(\partial \Omega)$. Then $u$ defined by

$u(x)=\inf _{\xi: \mathbb{R}^{+} \rightarrow A} \int_{0}^{t_{x}(\xi)} l\left(y_{x}(s), \xi(s)\right) d s+\varphi\left(y_{x}\left(t_{x}(\xi)\right)\right)$,

(where $y_{x}$ is the solution of the differential equation $y^{\prime}(t)=f(y(t), \xi(t)), t>0$, and $y(0)=x$, and where $t_{x}(\xi)$ is the first time the trajectory $y_{x}(., \xi)$ goes out of $\left.\bar{\Omega}\right)$ is a discontinuous viscosity solution of

$$
\left\{\begin{array}{lr}
H(x, \nabla u)=0 & \text { in } \Omega \\
u=\varphi & \text { on } \partial \Omega
\end{array}\right.
$$

The hypotheses (H6)-(H8) are:

(H6) $A$ is a compact topological space and $\Omega$ is a bounded open subset of $\mathbb{R}^{2}$;

(H7) $f: \bar{\Omega} \times A \rightarrow \mathbb{R}^{2}$ is continuous,

$l: \bar{\Omega} \times A \rightarrow \mathbb{R}$ is continuous and bounded; 
(H8) $f$ and $l$ are Lipschitz continuous in $x \in \bar{\Omega}$ uniformly in $a \in A$.

As we will see below, all the SFS Hamiltonians can be rewritten as supremums. The reader unfamiliar with control theory can read appendix A of [51] in which we detail the tools allowing to make this transformation. Finally, let us emphasize that, as shown by theorem 3 the existence of the discontinuous viscosity solution (with DBC) does not require anymore that $\varphi$ verifies the "compatibility condition".

\subsection{Hamiltonians for the SFS problems and unification of the "perspective" and "or- thographic SFS"}

\subsubsection{Hamiltonians for SFS}

In chapter 2, we have presented several PDEs arising from various mathematical formulations of the SFS problem. Let us recall that the definition of the viscosity solutions is associated with the Hamiltonians and not with the equations. Therefore for each SFS equation we have to specify a Hamiltonian.

With the Eikonal equation (6), we associate the Hamiltonian $H_{\text {Eiko }}^{\text {orth }}$ :

$$
H_{E i k o}^{\text {orth }}(x, p)=|p|-\sqrt{\frac{1}{I(x)^{2}}-1} .
$$

With equation (3), we associate the Hamiltonian $H_{R / T}^{\text {orth }}$ (introduced by Rouy and Tourin [55]):

$$
H_{R / T}^{\text {orth }}(x, p)=I(x) \sqrt{1+|p|^{2}}+p \cdot \mathbf{l}-\gamma .
$$

With equation (5), we associate the Hamiltonian $H_{D / O}^{\text {orth }}$ (introduced by Dupuis and Oliensis [17]):

$$
H_{D / O}^{\text {orth }}(x, p)=I(x) \sqrt{|p-\mathbf{l}|^{2}+\gamma^{2}}+p \cdot \mathbf{l}-1 .
$$

With equation (8) of the "perspective SFS" with a distant light source, we associate the Hamiltonian $H_{P / F}^{p e r s}$ (introduced by Prados and Faugeras [50]):

$$
\begin{aligned}
& H_{P / F}^{\text {pers }}(x, p)= \\
& I(x) \sqrt{f^{2}|p|^{2}+(x \cdot p+1)^{2}}-(f 1+\gamma x) \cdot p-\gamma ;
\end{aligned}
$$

and with the "perspective SFS" with a single point light source located at the optical center, we associate the Hamiltonian $H_{F}^{\text {pers }}$ :

$\left(Q(x)=f / \sqrt{|x|^{2}+f^{2}}\right)$

$$
\begin{aligned}
& H_{F}^{\text {pers }}(x, p)= \\
& \quad I(x) \sqrt{f^{2}|p|^{2}+(p \cdot x)^{2}+Q(x)^{2}}-Q(x) .
\end{aligned}
$$

\subsubsection{A "generic" Hamiltonian for SFS}

As we have seen in the previous section, the SFS problem leads to several Hamiltonians. Nevertheless we show that all these SFS Hamiltonians are special cases of a general one, thereby simplifying the formalization of the problem.

\section{Explicit formulation of the "generic SFS" Hamiltonian}

In [51], we show that all the SFS Hamiltonians $H_{*}^{\text {orth }}$ and $H_{*}^{\text {pers }}$ are special cases of the following "generic" Hamiltonian $H_{g}$ defined by:

$$
H_{g}(x, p)=\tilde{H}_{g}\left(x, A_{x} p+\mathbf{v}_{x}\right)+\mathbf{w}_{x} \cdot p+c_{x},
$$

with $\tilde{H}_{g}(x, q)=\kappa_{x} \sqrt{|q|^{2}+K_{x}^{2}}$ and where $\kappa(x)$, $K(x) \geq 0, A_{x}=D_{x} R_{x}, D_{x}=\left(\begin{array}{cc}\mu_{x} & 0 \\ 0 & \nu_{x}\end{array}\right), R_{x}$ is the rotation matrix $\frac{1}{|x|}\left(\begin{array}{cc}x_{2} & -x_{1} \\ x_{1} & x_{2}\end{array}\right)$ if $x \neq 0, R_{x}=I d_{2}$ if $x=0, \mu_{x}, \nu_{x} \neq 0, \mathbf{v}_{x}, \mathbf{w}_{x} \in \mathbb{R}^{2}$ and $c_{x} \in \mathbb{R}$.

The associated functions $\mathbf{v}_{x}, \mathbf{w}_{x}, c_{x}, \mu_{x}, \nu_{x}, \kappa_{x}$ and $K_{x}$, for the various SFS Hamiltonians are:

- for the "Rouy/Tourin Hamiltonian" $H_{R / T}^{\text {orth }}$ :

$$
\begin{aligned}
& \mu_{x}=1, \nu_{x}=1, \kappa_{x}=I(x), K_{x}=1, \\
& \mathbf{w}_{x}=1, \mathbf{v}_{x}=0, c_{x}=-\gamma ;
\end{aligned}
$$

- For the "Dupuis/Oliensis Hamiltonian" $H_{D / O}^{\text {orth }}$ :

$$
\begin{aligned}
& \mu_{x}=1, \nu_{x}=1, \kappa_{x}=I(x), K_{x}=\gamma, \\
& \mathbf{w}_{x}=1, \mathbf{v}_{x}=-R_{x} \mathbf{l}, c_{x}=-1 ;
\end{aligned}
$$

- For the "Eikonal Hamiltonian" $H_{\text {Eiko }}^{\text {orth }}$ :

$$
\begin{aligned}
& \mu_{x}=1, \nu_{x}=1, \kappa_{x}=1, K_{x}=0, \\
& \mathbf{w}_{x}=0, \mathbf{v}_{x}=0, c_{x}=-\sqrt{\frac{1}{I(x)^{2}}-1} ;
\end{aligned}
$$

- For the "Perspective SFS" with a point light source at infinity $H_{P / F}^{p e r s}$ :

$$
\mu_{x}=f, \nu_{x}=\sqrt{f^{2}+|x|^{2}}, \kappa_{x}=I(x),
$$




$$
\begin{aligned}
& K_{x}=\sqrt{\frac{f^{2}}{f^{2}+|x|^{2}}}, \mathbf{w}_{x}=-(f \mathbf{l}+\gamma x), \\
& \mathbf{v}_{x}=D_{x}^{-1} R_{x} x=\left(0, \frac{|x|}{\sqrt{f^{2}+|x|^{2}}}\right), c_{x}=-\gamma .
\end{aligned}
$$

- For the "Perspective SFS" with a point light source located at the focal center $H_{F}^{\text {pers }}$ :

$$
\begin{aligned}
& \mu_{x}=f, \nu_{x}=\sqrt{f^{2}+|x|^{2}}, \kappa_{x}=I(x), \\
& K_{x}=\sqrt{\frac{f^{2}}{f^{2}+|x|^{2}}}, \mathbf{w}_{x}=0, \mathbf{v}_{x}=0, c_{x}=-K_{x} ;
\end{aligned}
$$

For all the SFS Hamiltonians, we can remark that $\left({ }^{t} R_{x} A_{x}\right)^{-1},{ }^{t} R_{x} A_{x},{ }^{t} R_{x} \mathbf{v}_{x}, K_{x}, \mu_{x}$ and $\nu_{x}$ are continuous (therefore bounded if $\bar{\Omega}$ is compact), that $\mathbf{w}_{x}$ is Lipschitz continuous (therefore bounded if $\bar{\Omega}$ is compact), that $\mathbf{v}_{x}, \kappa_{x}$ are bounded and that $\kappa_{x}=I(x)$ and $c_{x}$ is Lipschitz continuous and bounded ${ }^{9}$.

We call "generic SFS" equation, equation associated with the "generic SFS" Hamiltonian: $\forall x \in \Omega$,

$$
\tilde{H}_{g}\left(x, A_{x} \nabla u(x)+\mathbf{v}_{x}\right)+\mathbf{w}_{x} \cdot \nabla u(x)+c_{x}=0 .
$$

This formulation considerably simplifies the analysis of the problem. All theorems about the characterization and the approximation of the solutions can be proved by using this generic SFS Hamiltonian. In particular, this formulation unifies the orthographic and perspective ${ }^{10}$ SFS problems. Also, from a practical point of view, a unique code can be used to numerically solve these various problems.

\section{Control formulation of the "generic SFS" Hamiltonian}

From a theoretical point of view as well as from a practical one, it is very interesting to formulate the SFS Hamiltonians, and so the "generic SFS" Hamiltonian, as a supremum:

$$
H_{g}(x, p)=\sup _{a \in \bar{B}_{2}(0,1)}\left\{-f_{g}(x, a) \cdot p-l_{g}(x, a)\right\}
$$

$\left(\bar{B}_{2}(0,1)\right.$ is the closed unit ball of $\left.\mathbb{R}^{2}\right)$. For example, such a formulation allows to apply the existence theorem 3 to the SFS problem. Also, in section 4, we show that it allows to design approximations schemes and numerical algorithms. Therefore it allows to compute numerical approximations of the viscosity solutions of the SFS PDEs.

\footnotetext{
${ }^{9}$ Except for $H_{\text {Eiko }}^{\text {orth }}$.

${ }^{10}$ Including our new model with the light source located at the optical center.
}

By using the Legendre transform and differential calculus, we show in [51] that we can rewrite the Hamiltonian $H_{g}$ as the supremum (25) with

$$
\begin{aligned}
& f_{g}(x, a)=-\left[\kappa_{x}{ }^{t} R_{x} D_{x} R_{x} \cdot a+\mathbf{w}_{x}\right] \\
& l_{g}(x, a)=-\left[K_{x} \kappa_{x} \sqrt{1-|a|^{2}}+\kappa_{x}\left({ }^{t} R_{x} \mathbf{v}_{x}\right) \cdot a+c_{x}\right]
\end{aligned}
$$

\subsection{Existence of viscosity solutions of the SFS problems}

\subsubsection{Existence of continuous viscosity solutions of the SFS problems}

In this section, we apply theorem 2 to prove the existence of continuous viscosity solutions of the SFS Hamiltonians. Let us remind the reader that all the properties proved for the "generic SFS" Hamiltonian are also available for all the SFS Hamiltonians.

- At first, the "generic SFS" Hamiltonian $H_{g}$ is convex with respect to $p$ : (H1) is true.

- About the uniform coercivity (hypothesis (H2) described in theorem 2), in [51] we prove the

Proposition 1 Let us consider the Hamiltonian $H_{g}$ (defined in section 3.3.2). Assume that $\kappa_{x}, c_{x},\left({ }^{t} R_{x} A_{x}\right)^{-1}, \mathbf{w}_{x},{ }^{t} R_{x} \mathbf{v}_{x}$ are continuous and bounded on the compact set $\bar{\Omega}$. If $\forall x \in \bar{\Omega}$, $\left|{ }^{t} A_{x}^{-1} \mathbf{w}_{x}\right|<\kappa_{x}$ then $H_{g}(x,$.$) is coercive uniformly { }^{11}$ with respect to $x$ in $\bar{\Omega}$.

Geometrically, this last condition holds iff the ambiguity cone (set of the unit vectors $\mathbf{n}$ verifying $\cos (\mathbf{n}, \mathbf{L})=$ $I(x)$ ) does not intersect the orthogonal plane to the projection line; see figure 5. Analytically, we obtain easily the following statements:

$\circ H_{E i k o}^{\text {orth }}, H_{F}^{\text {pers }}$ are uniformly coercive if $I(x)>0$.

$\circ H_{R / T}^{\text {orth }}, H_{D / O}^{\text {orth }}$ are uniformly coercive if $I(x)>|\mathbf{l}|$.

$\circ H_{P / F}^{\text {pers }}$ is uniformly coercive if

$$
I(x)^{2}>\frac{1}{f^{2}+|x|^{2}}\left[|\gamma x+f \mathbf{l}|^{2}+\left(|x|^{2}|\mathbf{l}|^{2}-(x \cdot \mathbf{l})^{2}\right)\right] .
$$

\footnotetext{
${ }^{11}$ The uniform coercivity property is the hypothesis $(\mathrm{H} 2)$ described in theorem 2
} 


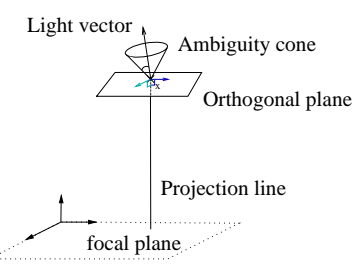

a) Orthographic case

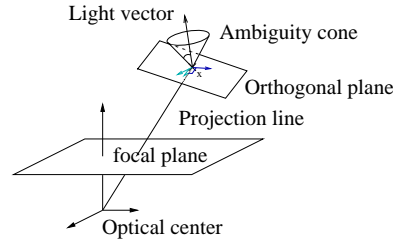

b) Perspective case
Figure 5: Ambiguity cone and plane orthogonal to the projection line.

Hence, subject to the adequate conditions ${ }^{12}$, all SFS Hamiltonians verify hypothesis (H2).

- Concerning hypothesis (H3), by taking the derivative, we verify that:

$$
\begin{aligned}
& \inf _{p \in \mathbb{R}^{2}} H_{g}(x, p)= \\
& \quad K_{x} \sqrt{\kappa_{x}^{2}-\left|{ }^{t} A_{x}^{-1} \mathbf{w}_{x}\right|^{2}}-\left({ }^{t} A_{x}^{-1} \mathbf{w}_{x}\right) \cdot \mathbf{v}_{x}+c_{x},
\end{aligned}
$$

if $\kappa_{x} \leq\left|{ }^{t} A_{x}^{-1} \mathbf{w}_{x}\right|$. Otherwise, $\inf _{p \in \mathbb{R}^{2}} H_{g}(x, p)=-\infty$. By substituting $K_{x}, \kappa_{x}, \mathbf{v}_{x}, \mathbf{w}_{x}, A_{x}$ by their adequate expressions, we prove that all the SFS Hamiltonians $H_{*}^{\text {orth }}$ and $H_{*}^{\text {pers }}$ verify (H3).

- Finally we prove that as soon as the intensity image $I$ is continuous ${ }^{13}$, all SFS Hamiltonians $H_{*}^{\text {pers }}$ and $H_{*}^{\text {orth }}$ are continuous in $\bar{\Omega} \times \mathbb{R}^{2}$.

Therefore, if the compatibility condition (H5) is satisfied on $\partial \Omega$ (if the intensity image is continuous and if the coercivity conditions are verified), then all the SFS problems (PDEs with DBC) have continuous viscosity solutions.

\subsubsection{Existence of discontinuous viscosity solutions of the SFS problems}

In section 3.3.2 we have rewritten the "generic SFS" Hamiltonian $H_{g}$ as the supremum:

$$
H_{g}(x, p)=\sup _{a \in \bar{B}_{2}(0,1)}\left\{-f_{g}(x, a) \cdot p-l_{g}(x, a)\right\} .
$$

$f_{g}$ and $l_{g}$ being detailed in section 3.3.2. In the report [51], we prove that as soon as the intensity image

\footnotetext{
${ }^{12}$ Let us note that, when the direction of the light is not too far from vertical and the brightness is not too dark (i.e. the ambiguity cone is relatively tight) this assumption generally holds in practice.

${ }^{13}$ For the Hamiltonian $H_{E i k o}^{\text {orth }}, I$ must also verify $I>0$ on $\bar{\Omega}$.
}

I is Lipschitz continuous, the hypotheses (H6)-(H8) hold for all SFS Hamiltonians ${ }^{14}$. Therefore, theorem 3 applies for each model of the SFS problem. Thus, for all $\varphi \in B C(\partial \Omega)$ there exists a discontinuous viscosity solution of all our SFS equations (PDEs with DBC). The compatibility conditions are no more required ${ }^{15}$.

\subsection{Characterization of the viscosity solu- tions of the SFS problems}

In the previous section we have proved the existence of viscosity solutions of the Lambertian SFS problems. Nevertheless, as we will show in this section, the SFS problem with DBC (on the boundary of the image $\partial \Omega$ ) do not have a unique viscosity solution. For computing a numerical solution of the SFS problems, we need to choose one solution among all. To make this choice, we must characterize the solutions. As Rouy and Tourin have proposed in [55] we achieve this goal by enlarging the DBC to the set $\partial \Omega \cup\{x \mid I(x)=1\}$.

\subsubsection{Uniqueness results for the continuous viscosity solutions of the SFS problem when $I(x)<1$}

The following theorem allows to prove the uniqueness of the continuous viscosity solution of the SFS equations when the intensity image $I$ does not reach the (maximal) value 1 . This uniqueness result ${ }^{16}$ is due to Ishii [28] and has been proved later in a different manner by Lions [41].

Theorem 4 (uniqueness) Let $\Omega$ be a bounded open subset of $\mathbb{R}^{2}$. Let us consider the equation

$$
H(x, \nabla u(x))=0 \quad \forall x \in \Omega .
$$

If $H$ verifies the hypotheses (H1), (H9) and (H10) (described below) then there exists at most one continuous viscosity solution u of (26), continuous in $\bar{\Omega}$, such that

$$
u(x)=\varphi(x), \forall x \in \partial \Omega .
$$

\footnotetext{
${ }^{14}$ For the Hamiltonian $H_{\text {Eiko }}^{\text {r th }}$, we also need to impose $I>0$ on $\bar{\Omega}$.

${ }^{15}$ Let us note that, also, the coercivity condition (H2) is no more required.

${ }^{16}$ For more general conditions, see [42]. A proof can be found in section II.5.3 of Bardi and Capuzzo-Dolcetta 's book [1].
} 
(H9) [space variable regularity] There exists a nondecreasing function $\omega$ which goes to zero at zero, such that $\forall x, y \in \Omega, \forall p \in \mathbb{R}^{N}, \quad|H(x, p)-H(y, p)| \leq$ $\omega(|x-y|(1+|p|))$.

(H10) [strict subsolution] there exists a strict viscosity subsolution $\underline{u} \in C^{1}(\Omega) \cap C(\bar{\Omega})$ of (26) (i.e. such that $H(x, \nabla \underline{u}(x))<0$ for all $x$ in $\Omega)$;

When the intensity image verifies $\forall x \in \Omega I(x)<1$, theorem 4 applies to all the SFS equations. In effect:

- the convexity of $H_{g}$ is clear: (H1) holds;

- for all the SFS Hamiltonians $H_{*}^{\text {orth }}$ and $H_{*}^{\text {pers }}$, the hypothesis (H9) is true as soon as the intensity image $I$ is Lipschitz continuous ${ }^{17}$;

- assuming that for all $x$ in $\Omega, I(x)<1$, the reader can verify that all constant functions are strict viscosity subsolutions of the Hamiltonians $H_{E i k o}^{\text {orth }}, H_{D / O}^{o r t h}$ and $H_{F}^{\text {pers }}$, that $\tilde{u}: x \longmapsto-\frac{1}{\gamma} \mathbf{l} \cdot x$ is a strict viscosity subsolution of the Hamiltonian $H_{R / T}^{\text {orth }}$, and that $\tilde{u}$ : $x \longmapsto-\ln \frac{\gamma}{f}-\ln (\gamma f-\mathbf{l} \cdot x)$ is a strict viscosity subsolution of the Hamiltonian $H_{P / F}^{\text {pers }}$ (we need to impose $\gamma f-\mathbf{l} \cdot x>0$, ie. $\mathbf{L} \cdot(x,-f)<0)$.

Thus, as soon as the intensity image $I$ is Lipschitz continuous and verifies

$$
\forall x \in \Omega, \quad I(x)<1,
$$

all the SFS equations (with DBC) have at most one continuous viscosity solution.

\subsubsection{Characterization of the continuous viscosity so- lutions of the SFS problem when $\{x \mid I(x)=$ $1\} \neq \emptyset$}

In practice, $I$ can reach the value 1 in an arbitrary compact set in $\bar{\Omega}$. This implies that there does not exist a strict viscosity subsolution and we lose uniqueness. In [55], Rouy and Tourin characterize the loss of uniqueness of the continuous viscosity solution of the equation

$$
\begin{cases}H(x, \nabla u(x))=0 & \forall x \in \Omega \\ u=\varphi & \forall x \in \partial \Omega,\end{cases}
$$

\footnotetext{
${ }^{17}$ For the Eikonal Hamiltonian $H_{E i k o}^{o r t h}$, we also need to impose $\forall x \in$ $\Omega, I(x)>0$.
}

in the case where $H$ is the Hamiltonian $H_{R / T}^{\text {orth }}$. We generalize their result to the continuous viscosity solutions of all our SFS Hamiltonians ( $H_{*}^{\text {orth }}$ and $H_{*}^{\text {pers }}$ ). In particular, we extend their work to the "perspective SFS" problem.

We denote $\mathcal{S}$ the set of singular points (also called critical points):

$$
\mathcal{S}=\{x \in \Omega \mid I(x)=1\} .
$$

In this work, we assume that $\mathcal{S}$ contains a finite number of isolated points, $\mathcal{S}=\left\{x_{1}, \ldots, x_{n}\right\}^{18}$. Let us fix $n$ real constants $\left(c_{i}\right)_{i=1 . . n}$. Thanks to the result presented in the previous section, we can claim that, as soon as the intensity image $I$ is Lipschitz continuous, all SFS equations (27) with DBC have at most one continuous viscosity solution $u$ such that for all $i=1 . . n, u\left(x_{i}\right)=c_{i}$. To prove this last assertion, we just have to enlarge the DBC to the set $\partial \Omega \cup \mathcal{S}$ and to apply theorem 4 . Thus, for characterizing a SFS continuous viscosity solution, we can ignore the set of singular points $\mathcal{S}$ and work in the open set $\Omega^{\prime}=\Omega-\mathcal{S}$. Therefore, we consider the problem

$$
\begin{cases}H(x, \nabla u(x))=0 & \forall x \in \Omega^{\prime} \\ u(x)=\varphi(x) & \forall x \in \partial \Omega^{\prime},\end{cases}
$$

rather than (27). So, by using the existence result of section 3.4.1, we prove that, if the intensity image $I$ is Lipschitz continuous (and if the coercivity and compatibility conditions are verified), then for all SFS equations (28), there exists a unique continuous viscosity solution. Thus, all the continuous viscosity solutions of (27) are then obtained from these by choosing almost arbitrarily ${ }^{19}$ the constants $c_{i}\left(=\varphi\left(x_{i}\right)\right)$.

In practice, for computing a numerical solution of the SFS problem, we must characterize the solution we want to compute, first. The characterization we propose here is somewhat disappointing. In effect, it assumes that we know the values of the solution at all the singular points and on the boundary of the image. But the input data to a

\footnotetext{
${ }^{18}$ The situations where $\stackrel{\circ}{\mathcal{S}}$ (the interior of the set $\mathcal{S}$ ) is not empty, are non generic. In effect, for a given experimental setup (surface, light, camera) such that $\stackrel{\circ}{\mathcal{S}} \neq \emptyset$, an arbitrarily small change in the experimental parameters (for example, when the light moves) will make $\stackrel{\circ}{\mathcal{S}}=\emptyset$. An image such that $\stackrel{\circ}{\mathcal{S}} \neq \emptyset$ is highly unlikely.

${ }^{19}$ Let us recall that for ensuring the existence of a continuous viscosity solution, the compatibility condition must be verified.
} 
SFS problem consists only in general of an image. We do not have at our disposal the values of the solution at the singular points or on the boundary of the image. Nevertheless, although this may appear a bit restrictive, in this article we will assume that we know these "boundary" data. In a forthcoming paper, we will describe how to remove this constraint. Another possibility is to choose among all solutions one which possesses an extra property, as in the work of Falcone et al. [8, 22, 23] where the uniqueness is obtained by choosing the maximal solution. The work of Falcone is based on the notion of "singular viscosity solutions". This notion pioneered by Ishii and Ramaswamy [31], has been recently upgraded by Camilli $[9,7]$. Let us emphasize that in his work Falcone assumes (as we do) that the solution is known on the boundary $\partial \Omega$.

\subsubsection{Case of the discontinuous viscosity solutions}

The uniqueness results for the discontinuous viscosity solutions are almost the same as the uniqueness results for the continuous viscosity solutions. Nevertheless, they need stronger hypotheses; which is reasonable because discontinuous viscosity solutions are weaker solutions than continuous viscosity solutions (the set of the discontinuous viscosity solutions of an equation contains the set of the continuous viscosity solutions). In particular, in the discontinuous case, in order to have uniqueness we need a strong uniqueness property (see section 2.2.3 of [54]). Let us remind that, in the framework of the disconstinuous viscosity solutions, we consider the PDE

$$
F(x, u(x), \nabla u(x))=0, \quad \forall x \in \bar{\Omega} ;
$$

where $F$ be a function (defined as in section 3.2.2) which takes into account the boundary data.

Definition 4 Let $\Omega$ be an open subset of $\mathbb{R}^{2}$, let $E \subset \bar{\Omega}$ and let $F$ be a function defined as in section 3.2.2. We say that the strong uniqueness property holds on the set E for the equation (29) when we have: "for all subsolution $u$, for all supersolution $v$ and for all $x$ in $E, u(x) \leq v(x)$ ”.

We have the following strong uniqueness result ${ }^{20}$ :

\footnotetext{
${ }^{20}$ See theorem 4.5 (and more exactly its corollary 4.1 ) of Barles'book [2] in the particular case where the Hamiltonian $H$ does not depend on $u$.
}

Theorem 5 Let $\Omega$ be smooth enough, let $H$ and $\varphi$ be two continuous functions defined as in section 3.2.2. If $H$ satisfies the hypotheses (H1), (H9) and (H10') ${ }^{21}$, and if $H$ satisfies the boundary hypotheses $(\mathrm{H} 11)$ and $(\mathrm{H} 12)$ which impose properties of $H$ on $\partial \Omega$, then the strong uniqueness property holds on the set $\Omega$ for the equation (29), where $F$ is defined as in section 3.2.2.

The hypothesis (H10') is a hypothesis slightly stronger than hypothesis $(\mathrm{H} 10)$ of the theorem 4 :

(H10') [strict subsolution] there exist $\underline{u} \in C^{1}(\Omega) \cap$ $C(\bar{\Omega})$ and $\delta<0$ such that $\forall x \in \Omega, H(x, \bar{\nabla} \underline{u}(x))<\delta$.

The hypotheses (H11) and (H12) are the following: there exist a neighborhood $\Gamma$ of $\partial \Omega$ (ie. $\Gamma$ is an open subset of $\mathbb{R}^{2}$ s.t. $\left.\partial \Omega \subset \Gamma\right)$ such that

(H11) $[p$ - regularity on $\partial \Omega]$ There exists a function $\omega$ which goes to zero at zero, such that $\forall x \in \Gamma, \forall p, q \in \mathbb{R}^{N}$, $|H(x, p)-H(x, q)| \leq \omega(|p-q|)$;

(H12) [coercivity] $H(x, p)$ coerciv in $p$ uniformly with respect to $x$ in $\Gamma$.

Clearly the strong uniqueness property involves the uniqueness of the discontinuous viscosity solution. Therefore, thanks to theorem 5, we can prove the uniqueness of the discontinuous viscosity solution of (29) in $\Omega$. Note that generally we do not have uniqueness in $\bar{\Omega}$.

Theorem 5 applies to the SFS problem. The three hypotheses (H1), (H9) and (H10') are almost the same as the hypotheses of theorem 4. As in the previous section we can prove that they are verified for the SFS Hamiltonians $H_{*}^{\text {orth }}$ and $H_{*}^{\text {pers }}$ as soon as the intensity image is Lipschitz continuous and verifies $I<1$ in $\bar{\Omega}$. Concerning the hypothesis (H11), we can prove that it holds for all SFS Hamiltonians $H_{*}^{\text {orth }}$ and $H_{*}^{\text {pers }}$. Moreover, let us remind that, in section 3.4.1, we have detailed the conditions involving the coercivity of all the SFS Hamiltonians. Therefore, if the intensity image $I$ is Lipschitz continuous, if $I$ verifies $I<1$ on $\bar{\Omega}$ and if the values of $I$ on the boundary of the image are such that the coercivity hypothesis holds, then there exists at most one discontinuous viscosity solution in $\Omega$.

\footnotetext{
${ }^{21}$ Note that these hypotheses are very close to the hypotheses of the uniqueness theorem 4
} 
Contrary to the continuous case, the above result (the uniqueness of the discontinuous viscosity solution of the SFS problem) does not apply on the set $\Omega^{\prime}=\Omega-\mathcal{S}$ (when the set $\mathcal{S}$ of the singular points is not empty). The reason of this lies on the difference between the hypotheses (H10) and (H10'). The uniqueness of the continuous viscosity solution only requires the hypothesis (H10) (theorem 4), whereas the uniqueness of the discontinuous viscosity solution requires the stronger hypothesis (H10') (theorem 5). In the first case, the hypothesis (H10) holds even if there are singular points on the boundary of $\Omega$, whereas in the second case, the hypothesis (H10') imposes that $\forall x \in \bar{\Omega}, I(x)<1$; hence there cannot be any singular points in $\partial \Omega$. Note that the hypothesis (H10') is optimal for obtaining the uniqueness of the discontinuous viscosity solution, see [51], for an illustration of this fact by considering the particular case of the Eikonal equation. As a matter of fact, this limitation is not really a problem. In effect, in the previous case we have assumed that we knew the values of the solution at all the singular points of the image. It is not more absurd to assume that we know the values of the solution in an arbitrarily small neighbourhood of the set the singular points. Thus, for characterizing a discontinuous viscosity solution, we can specify its values on the boundary of the image and in a neighbourhood of its critical points.

\subsection{Noise robustness of the viscosity solu- tions of SFS}

In computer vision or more generally in image processing, the images are always corrupted by noise. It is therefore very important to design schemes and algorithms robust to noise. That is to say we would like that the result obtained by the algorithm from a noisy image be close to the ideal result obtained from the perfect image. This property is often difficult to guarantee. For the "SFS" problem, the robustness is mathematically expressed by the continuity of the application which, given an image $I$, returns the associated surface $u$. In other words, we would like that, for all sequences of noisy images $I_{\varepsilon}$ uniformly converging toward an image $I$, the sequence of recovered solutions $u_{\varepsilon}$ uniformly converges toward the solution $u$ associated to $I$. In the research report [54], section 4.1.3, we have proved that if the intensity image $I$ verifies
$I(x)<1$ for all $x$ in $\bar{\Omega}$, then the viscosity solutions of the orthographic SFS problem (associated to the Hamiltonian $\left.H_{R / T}^{o r t h}\right)$ are robust to noise. This also applies to the other SFS Hamiltonians $H_{*}^{\text {orth }}$ and $H_{*}^{\text {pers }}$. In effect, the reader will verify that the proof proposed for the orthographic case can be adapted to the generic Hamiltonian.

In the same idea, it is possible to prove that the viscosity solutions of the SFS problems are robust with respect to inaccuracies in light and focal parameters. Nevertheless, the proof of this statement requires much more sophisticated tools and will be the concern of another furthcoming paper.

\section{Two approximation schemes for the "generic SFS" equation}

In section 3.3.2, we have shown that the various models of the Lambertian SFS problems can be unified by the "generic SFS" Hamiltonian $H_{g}$. In this section, we present two schemes approximating the "generic SFS" equation (24). These approximation schemes allow to solve numerically this equation. Let us note that the numerical method we present here can be generalized to all Hamilton-Jacobi-Bellman equations, see [51].

\subsection{Approximation schemes}

In this section, we remind the reader of the definition of an approximation scheme. An approximation scheme is a functional equation of the form

$$
T\left(\rho, x, u^{\rho}\right)=0 \quad \forall x \in \bar{\Omega} ;
$$

where $T: \mathcal{M} \times \bar{\Omega} \times B(\bar{\Omega}) \rightarrow \mathbb{R}, \mathcal{M}=\mathbb{R}^{+} \times \mathbb{R}^{+}$, and $B(D)$ is the space of bounded functions defined on a set $D . \rho \in \mathcal{M}$ defines the size of the mesh that is used in the corresponding numerical algorithms, see section 5, $u^{\rho}$ is a solution of the scheme $T$. For $h_{1}, h_{2} \in \mathbb{R}^{+}$, we write $\rho=\left(h_{1}, h_{2}\right)$. If $h_{1}=h_{2}$, we let $\rho=h_{i} \in \mathbb{R}^{+}$. Also, we (mis)use the notation " $\forall \rho>0$ " which stands for " $\forall \rho \in \mathcal{M}$ such that $h_{1}>0$ and $h_{2}>0 "$.

Following [3], we introduce the representations $S$ of a scheme $T$ as

$$
S\left(\rho, x, u^{\rho}(x), u^{\rho}\right)=0 \quad \forall x \in \bar{\Omega}
$$


where

$$
\begin{aligned}
S: \mathcal{M} \times \bar{\Omega} \times \mathbb{R} \times B(\bar{\Omega}) & \longrightarrow \mathbb{R} \\
(\rho, x, t, u) & \longmapsto S(\rho, x, t, u) .
\end{aligned}
$$

Note that a representation of a scheme is also a scheme. It is a way to simplify computations. In effect, the representation of a scheme $T\left(\rho, x, u^{\rho}\right)=0$ by a scheme of the form $S\left(\rho, x, u^{\rho}(x), u^{\rho}\right)=0$ suggests an iterative algorithm for computing a numerical approximation of the solution of the scheme. Given $u^{n}$ (the approximation of $u^{\rho}$ at step $n$ ), and a point $x$ of $\bar{\Omega}$, the associated algorithm consists in solving the equation

$$
S\left(\rho, x, t, u^{n}\right)=0
$$

with respect to $t$. A solution of (30) is the updated value of $u^{n}$ at $x$ (see section 5 ). When this solution can be obtained explicitely we talk about explicit schemes, when it cannot, we talk about implicit schemes, see next section.

In the SFS problem, the open set $\Omega$ is bounded. In practice, we generally consider the rectangular domain $] 0, X[\times] 0, Y\left[\right.$ of $\mathbb{R}^{2}$. Since we are considering the "generic SFS" equation with Dirichlet boundary conditions, we consider "schemes with Dirichlet boundary conditions". These schemes are defined by $S\left(\rho, x, u^{\rho}(x), u^{\rho}\right)=0$, where $S$ is defined by

$$
S(\rho, x, t, u)= \begin{cases}\tilde{S}(\rho, x, t, u) & \text { if } x \in \Omega^{\rho}, \\ t-\varphi(x) & \text { if } x \in b \Omega^{\rho},\end{cases}
$$

where $\Omega^{\rho}=\left\{x \in \Omega \mid x \pm h_{1} \overrightarrow{e_{1}} \in \bar{\Omega}\right.$ and $\left.x \pm h_{2} \overrightarrow{e_{2}} \in \bar{\Omega}\right\}$, and $b \Omega^{\rho}=\bar{\Omega}-\Omega^{\rho}$. Since $\varphi$ is defined only on $\partial \Omega$, we assume in (31) that we have extended it continuously to $b \Omega^{\rho}$. We now introduce the

Definition 5 (monotonicity) The

scheme $S\left(\rho, x, u^{\rho}(x), u^{\rho}\right)=0$ defined in $\bar{\Omega}$, is monotone if $\forall \rho \in \mathcal{M}, \forall x \in \bar{\Omega}, \forall t \in \mathbb{R}$ and $\forall u, v \in B(\bar{\Omega})$,

$$
u \leq v \quad \Longrightarrow \quad S(\rho, x, t, u) \geq S(\rho, x, t, v)
$$

(the scheme is nonincreasing with respect to $u$ )

There exists essentially only one method for proving the convergence of the solutions of schemes toward viscosity solutions, i.e. the one presented by Barles and Souganidis in [3]. This method requires the monotonicity of the scheme; this is why we design monotone schemes in the sequel.

\subsection{Two "generic SFS" approximation schemes}

\subsubsection{An "implicit" scheme}

Let us remind the reader that in section 3.3.2, we have rewritten the "generic SFS" equation as a supremum:

$$
\sup _{a \in B(0,1)}\left\{-f_{g}(x, a) \cdot \nabla u(x)-l_{g}(x, a)\right\}=0 \quad \forall x \in \Omega .
$$

For lighter notations, we denote $f_{1}(x, a)$ (respectively $f_{2}(x, a)$ ) the first (respectively, the second) component of $f_{g}(x, a)$. In this section, we design an approximation scheme of (32) by using only the backward and forward approximations of the partial derivatives. Thus in order to guarantee the monotonicity of the scheme, it appears natural to replace $\partial_{\overrightarrow{e_{i}}} u(x)$ with $\left(\frac{t-u\left(x-h_{i} \overrightarrow{e_{i}}\right)}{h_{i}}\right)$ when $-f_{i}(x, a) \geq 0$ and by $\left(\frac{u\left(x+h_{i} \overrightarrow{e_{i}}\right)-t}{h_{i}}\right)$ when $-f_{i}(x, a) \leq$ 0 . We therefore consider the scheme $S$ with $\tilde{S}$ (see equation (31)) defined as

$$
\begin{aligned}
& \tilde{S}(\rho, x, t, u)= \\
& \sup _{a \in \bar{B}(0,1)}\left\{-f_{1}(x, a)\left(\frac{t-u\left(x+s_{1}(x, a) h_{1} \overrightarrow{e_{1}}\right)}{-s_{1}(x, a) h_{1}}\right)\right. \\
& \left.-f_{2}(x, a)\left(\frac{t-u\left(x+s_{2}(x, a) h_{2} \overrightarrow{e_{2}}\right.}{-s_{2}(x, a) h_{2}}\right)-l_{g}(x, a)\right\} \\
& =\sup _{a \in \bar{B}(0,1)}\left\{-f_{g}(x, a) \cdot D(\rho, x, t, u, a)-l_{g}(x, a)\right\},
\end{aligned}
$$

where $s_{i}(x, a)$ is the sign of $f_{i}(x, a)$ and $D(\rho, x, t, u, a)$ is an approximation of the gradient: $D(\rho, x, t, u, a)=$ $\left(\frac{t-u\left(x+s_{1}(x, a) h_{1} \overrightarrow{e_{1}}\right)}{-s_{1}(x, a) h_{1}}, \frac{t-u\left(x+s_{2}(x, a) h_{2} \overrightarrow{e_{2}}\right)}{-s_{2}(x, a) h_{2}}\right)$. The function $\tilde{S}$ defined by (33) is clearly nondecreasing with respect to $t$ and nonincreasing with respect to $u$. Therefore the scheme $S$ with $\tilde{S}$ defined by (33) is monotone. In section 4.3 , we prove that this scheme is stable. Since the variable $t$ appears inside the sup operator, the scheme is implicit ${ }^{22}$.

\footnotetext{
${ }^{22}$ Let us note that by using (differential and algebric) calculus, we can express explicitly the solution of the equation in $t, \tilde{S}(\rho, x, t, u)=0$. This step is necessary for implementing the associated algorithm.
} 


\subsubsection{A "Semi implicit" scheme}

A classical method to deal with the implicit scheme (33) consists in transforming the scheme into a fixed point problem. We multiply $\tilde{S}$ by a fictitious time increment $-\Delta \tau$ (with $\Delta \tau>0$ ) and we add $u^{\rho}(x)$ to both sides of the equation $\tilde{S}=0$. In other words, instead of considering the scheme defined by $\tilde{S}(\rho, x, t, u)$, we consider the one defined by the function

$$
\tilde{S}_{2}(\rho, x, t, u)=t-u(x)+\Delta \tau \tilde{S}(\rho, x, u(x), u) .
$$

For the sake of simplicity, we write $s_{i}$ for $s_{i}(x, a)$ in the sequel. Thus we obtain a new formulation of the scheme $S\left(\rho, x, u^{\rho}(x), u^{\rho}\right)=0$ by defining

$$
\begin{gathered}
\tilde{S}_{2}(\rho, x, t, u)=t-u(x)+\Delta \tau \\
\sup _{a \in \bar{B}(0,1)}\left\{-f_{g}(x, a) \cdot D(\rho, x, u(x), u, a)-l_{g}(x, a)\right\}, \\
=t+\sup _{a \in \bar{B}(0,1)}\left\{-\left[1-\Delta \tau\left(\Lambda_{1}+\Lambda_{2}\right)\right] u(x)\right. \\
-\Delta \tau\left[\Lambda_{1} u\left(x+s_{1} h_{1} \overrightarrow{e_{1}}\right)+\Lambda_{2} u\left(x+s_{2} h_{2} \overrightarrow{e_{2}}\right)\right] \\
\left.-\Delta \tau l_{g}(x, a)\right\} ;
\end{gathered}
$$

where $\Lambda_{i}=\frac{\left\lfloor f_{i}(x, a)\right\rfloor}{h_{i}}$. Note that $\tilde{S}_{2}(\rho, x, t, u)$ is nondecreasing with respect to $t$ and nonincreasing with respect to $u$ as soon as the function $\xi \mapsto-\xi+\Delta \tau \widetilde{S}(\rho, x, \xi, u)$ is nonincreasing. Also, we can verify easily that the scheme associated to (34) is monotone iff $\Delta \tau$ is small enough $\left(\Delta \tau \leq\left(\Lambda_{1}+\Lambda_{2}\right)^{-1}\right.$, for all $a$ in $A$ and for all $x$ such that $f(x, a) \neq 0$. If $f(x, a)=0$, no constraints are required). In other words, this formulation of the decentered schemes requires that some conditions be satisfied in order to be monotone. Despite this disadvantage, the formulation (34) is interesting because it yields semi implicit algorithms whereas the formulation (33) provides totally implicit algorithms. We use the expression "semi implicit" because the value of the sup has to be evaluated at each point $x$, but it does not involve $t$. Nevertheless, we will see that the algorithms resulting from the formulation (33) can be made explicit through the use of calculus.

Remark 1 Let us mention that the larger the "parameter" $\Delta \tau$, the faster the convergence. Therefore, if $f\left(x, a_{0}\right) \neq 0$ (where $a_{0}$ is the optimal control of (34)), we can choose an optimal $\Delta \tau$ :

$$
\Delta \tau_{\text {opt }}=\left(\Lambda_{1}+\Lambda_{2}\right)^{-1} .
$$

Let us remark that $a_{0}$ and the optimal $\Delta \tau_{\text {opt }}$ depend on $x$, but that $a_{0}$ does not depend on $\Delta \tau$. Thus, for all $x$ such that $f\left(x, a_{0}\right) \neq 0$, if we choose $\Delta \tau=\Delta \tau_{\text {opt }}$, the scheme (34) becomes:

$$
\begin{aligned}
& \tilde{S}_{2}^{\text {opt }}(\rho, x, t, u)=t \\
& \quad-\sum_{i=1}^{2} \frac{\Lambda_{i}}{\Lambda_{1}+\Lambda_{2}} u\left(x+s_{i} h_{i} \overrightarrow{e_{i}}\right)-\frac{l_{g}\left(x, a_{0}\right)}{\Lambda_{1}+\Lambda_{2}},
\end{aligned}
$$

where $a_{0}$ is the optimal control of (34). The particular case where $f_{g}\left(x, a_{0}\right)=0$ is described in [51].

Let us emphasize the fact that the schemes defined by (34) have exactly the same solutions as those defined by (33).

\subsection{Stability of the "generic SFS" approxi- mation schemes}

In this section, we show that the (implicit and semiimplicit) "generic SFS" approximation schemes have always solutions. Note that all the results we present in this section are proved in [51] and that we have generalized them to the Hamilton-Jacobi-Bellman equations. We start with the definition of the stability of a scheme (according to Barles and Souganidis [3]).

Definition 6 (stability) The scheme $T\left(\rho, x, u^{\rho}\right)=0 \mathrm{de}$ fined on $\bar{\Omega}$, is stable if $\forall \rho>0$, it has a bounded solution $u^{\rho}$. It is uniformly stable if its solutions $u^{\rho}$ are bounded independently of $\rho$.

Note that the semi-implicit scheme (34) is stable (respectively, uniformly stable) iff the implicit scheme (33) is stable (respectively, uniformly stable). We prove the stability of the implicit "generic SFS" approximation scheme, by using a result based on the notion of the subsolutions of a scheme:

Definition 7 (subsolution of an approximation scheme) For a fixed $\rho>0, v^{\rho}: \bar{\Omega} \rightarrow \mathbb{R}$ is a subsolution of the scheme $T\left(\rho, x, u^{\rho}\right)=0$ if $\forall x \in \bar{\Omega}, T\left(\rho, x, v^{\rho}\right) \leq 0$.

This definition in hand, we can formulate proposition 2.

Proposition 2 Suppose that $\forall \rho>0$, there exists a subsolution of the scheme and that there exists $M^{\rho} \in \mathbb{R}$ such that for all subsolutions $v^{\rho}, \forall x \in \bar{\Omega}, v^{\rho}(x) \leq M^{\rho}$. If $f_{g}$ 
verifies the hypothesis (H13) (see below), then the implicit "generic SFS" scheme is stable. Moreover, if $M^{\rho} \in \mathbb{R}$ does not depend on $\rho$ then the stability is uniform.

(H13) For all $x \in \Omega$, there exists $a_{x} \in \bar{B}(0,1)$ such that $f_{g}\left(x, a_{x}\right) \neq 0$.

About the hypothesis (H13), we can remark that ( $x$ being fixed):

$$
\forall a \in \bar{B}(0,1), f_{g}(x, a)=0 \Longleftrightarrow \mathbf{w}_{x}=0 \text { and } \kappa_{x}=0 .
$$

In practice, for $H_{E i k o}^{\text {orth }}$ and $H_{F}^{\text {pers }}$, and for $H_{P / F}^{\text {pers }}, H_{R / T}^{\text {orth }}$ and $H_{D / O}^{\text {orth }}$ with $\mathbf{l}=0$, there are no shadows, therefore, $I(x)$ is (should be) never null. If $\mathbf{l} \neq 0$ then $\mathbf{w}_{x}$ associated with $H_{R / T}^{\text {orth }}$ and $H_{D / O}^{\text {orth }}$ is not null. For $H_{P / F}^{\text {pers }}$, it holds iff $I\left(-\frac{f}{\gamma} \mathbf{l}\right) \neq 0$.

This shows that the difficulties for proving the stability of the implicit "generic SFS" scheme lies in the proof of the existence of subsolutions and in the proof that the subsolutions are bounded. In the case where the Hamiltonian $H_{g}$ verifies $H_{g}(x, 0) \leq 0$, the following proposition ensures the existence of a subsolution:

Proposition 3 Let $\varphi$ be a bounded function defined on a neighbourhood of $\partial \Omega$. Iffor all $x$ in $\bar{\Omega}, H_{g}(x, 0) \leq 0$ then all constant functions $u$ on $\bar{\Omega}$ such that $u \leq \min _{x} \varphi(x)$, are subsolutions of the implicit "generic SFS" equation.

Clearly, this last proposition applies to $H_{\text {Eiko }}^{\text {orth }}, H_{F}^{\text {pers }}$ and $H_{D / O}^{\text {orth }}$. It also applies to $H_{R / T}^{\text {orth }}$ and $H_{P / F}^{\text {pers }}$ when $\mathbf{L}=(0,0,1)$. Concerning the general case of the Hamiltonian $H_{R / T}^{\text {orth }}$, we prove that $u_{0}(x):=-\frac{1}{\gamma} \mathbf{l} \cdot x+C$ (where $C$ is chosen such that $\left.\forall x \in \bar{\Omega}, u_{0}(x) \leq \min _{x} \varphi(x)\right)$ is a subsolution. We have not found subsolutions associated with the Hamiltonian $H_{P / F}^{\text {pers } 23}$

Finally, for proving that all the subsolutions of the "generic SFS" approximation scheme are upper bounded, we can use proposition 4 .

Notation 1 Let us remind the reader that for $i=1,2$, we note $f_{i}(x, a)$ the $i^{\text {th }}$ component of $f_{g}(x, a)$.

\footnotetext{
${ }^{23}$ As mentioned in [51], we can design another Hamiltonian (denoted $H_{2}^{\text {pers }}$ ) associated with the "perspective" equation (8) and verifying $H_{2}^{\text {pers }}(x, 0) \leq 0$.
}

Proposition 4 Suppose that for all $x$ in the bounded subset $\bar{\Omega}$, there exists a control $a_{x} \in B(0,1)$ such that for all $i=1,2$, the sign of $f_{i}\left(x, a_{x}\right)$ does not depend on $x$. For $i=1,2$, let us denote $s_{i}$ the sign of $f_{i}\left(x, a_{x}\right)$. Also, let us suppose that there exists $\varepsilon>0$ and $j$ in $\{1,2\}$ such that $\forall x \in \bar{\Omega}, s_{j} f_{j}\left(x, a_{x}\right) \geq \varepsilon$. If $l_{g}$ and $\varphi$ are upper bounded then all the subsolutions of the implicit "generic SFS" approximation scheme are upper bounded. Also, there exists $\mathrm{B}>0$ such that $\forall \rho \in \mathbb{R}$, for all subsolution $v_{\rho}$ of the implicit "generic SFS" approximation scheme, we have $v_{\rho} \leq \mathrm{B}$.

For all $x$ in $\bar{\Omega}$, let us $\operatorname{consider}^{24} a_{x}=$ $\operatorname{Dil}_{x}^{-1}\left[\left(\begin{array}{l}1 \\ 1\end{array}\right)-\mathbf{w}_{x}\right]$, then we have $f_{g}\left(x, a_{x}\right)=$ $-(1,1)$. Therefore proposition 4 applies as soon as $l_{g}$ is bounded (which holds for all SFS Hamiltonians because $\bar{\Omega}$ is bounded).

Therefore, proposition 2 applies to all SFS Hamiltonians ${ }^{25}$ implying that the implicit (and therefore semiimplicit) schemes are uniformly stable (for $\rho$ in $\mathbb{R}$ ).

Remark 2 Propositions 2, 3 and 4 do not require regularity with respect to the space variable $x$. In other words, the continuity of the intensity image I is not required for obtaining the stability of our SFS schemes. They are still relevant when the intensity image is discontinuous and when there are black shadows.

\subsection{Convergence toward the viscosity solu- tions of the "generic SFS" equation}

For proving the convergence of the solutions of an approximation scheme toward the viscosity solution of a Hamilton-Jacobi equation, we use the method (based on the notion of weak limits) due to Barles and Souganidis [3]. Remember that in the framework of discontinuous viscosity solutions, the PDE with Dirichlet boundary conditions is rewritten as:

$$
F(x, u(x), \nabla u(x))=0, \quad \forall x \in \bar{\Omega} ;
$$

where $F$ is defined on $\bar{\Omega} \times \mathbb{R} \times \mathbb{R}^{N}$ by

$$
F(x, u, p)= \begin{cases}H(x, p) & \text { if } x \text { in } \Omega \\ u-\varphi(x) & \text { if } x \text { on } \partial \Omega\end{cases}
$$

\footnotetext{
${ }^{24}$ We assume that $\forall x \in \bar{\Omega}, \kappa_{x} \neq 0$.

${ }^{25}$ Except for $H_{P / F}^{\text {pers }}$, because we have not found subsolutions of the associated scheme.
} 
We now give the definition of the consistency of an approximation scheme according to Barles and Souganidis [3] (in this section, we assume that $\rho \in \mathbb{R}$ ).

\section{Definition 8 (consistency)}

The scheme $S\left(\rho, x, u^{\rho}(x), u^{\rho}\right)=0$ is consistent with equation (35) if $\forall x \in \bar{\Omega}$ and $\forall \phi \in C_{b}^{\infty}(\bar{\Omega})$

and $\lim \sup \frac{S(\rho, y, \phi(y)+\xi, \phi+\xi)}{\rho} \leq F^{*}(x, \phi(x), \nabla \phi(x))$

$$
\liminf \frac{S(\rho, y, \phi(y)+\xi, \phi+\xi)}{\rho} \geq F_{*}(x, \phi(x), \nabla \phi(x))
$$

(where the limsup and liminf are taken when $\rho \rightarrow 0$, $y \rightarrow x$ and $\xi \rightarrow 0)$.

We recall that $F^{*}$ and $F_{*}$ are defined in section 3.2.2. In [51], we formulate a sufficient condition involving the consistency of the implicit "generic SFS" approximation scheme (with DBC) with the "generic SFS" equation (24) with DBC:

Proposition 5 If $f_{g}$ and $l_{g}$ verify the hypotheses (H6)(H8), then the implicit "generic SFS" approximation scheme (with DBC) is consistent with the "generic SFS" equation with DBC (equation (35) with $H=H_{g}$ ).

In the report [51], we show that the hypotheses (H6)-(H8) hold for all SFS Hamiltonians as soon as the intensity image I is Lipschitz continuous ${ }^{26}$. Moreover, we also prove there the following theorem:

Theorem 6 (convergence toward the viscosity solution) Let $S$ be a monotone, uniformly stable and consistent (with equation (35)) approximation scheme. Let us suppose that the strong uniqueness property is verified on a subset $D$ of $\bar{\Omega}$. Then the solutions $u^{\rho}$ of the scheme $S$ converge on $D$ toward the discontinuous viscosity solution of (35) when $\rho \rightarrow 0$.

By construction, the implicit "generic SFS" approximation scheme is monotone. In the previous section, we have proved that for all SFS Hamiltonians the associated scheme is uniformly stable (proposition 4 and 2). Also, they are consistent as soon as the intensity image $I$ is Lipschitz continuous (proposition 5). Finally, we have shown in section 3.5.3 that the strong uniqueness property holds as soon as ${ }^{27}$ the Hamiltonian is coercive with respect to $p$

\footnotetext{
${ }^{26}$ For the Hamiltonian $H_{E i k o}^{\text {orth }}$, we also need to impose $I>0$ on $\bar{\Omega}$.

${ }^{27}$ Let us recall that we assume that $\bar{\Omega}$ does not have critical points; see the end of section 3.5 .3 .
}

(uniformly with respect to $x$ ) in a neighborhood of $\partial \Omega$ (see section 3.4.1 for a description of the coercivity condition for all SFS Hamiltonians).

Therefore, subject to these last conditions, the solutions of the implicit SFS schemes converge toward the unique viscosity solution of the adequate SFS equation when $\rho \rightarrow 0$.

Remark 3 Since the solutions of the semi-implicit scheme are the same as the solutions of the implicit scheme, the convergence toward the viscosity solution also holds for the solutions of the semi-implicit scheme.

\section{Numerical algorithms for the "generic SFS" problem}

In section 4, we have designed two monotone schemes (an implicit and a semi-implicit one) approximating the "generic SFS" equation (24). We have proved the stability of these schemes. We have also described sufficient conditions ensuring the convergence of the solutions of the schemes toward the unique viscosity solution of the considered SFS equation. Let us remind the reader that the solutions of the implicit "generic SFS" approximation scheme are the same as the solutions of the semi-implicit one. We are now going to describe two iterative algorithms (an algorithm associated with the implicit "generic SFS" approximation scheme and another associated with the semi-implicit one) that compute some numerical approximations of a solution $u^{\rho}$ of our schemes (for a fixed $\rho>0)$.

Let us fix $\rho>0 ; \rho=\left(h_{1}, h_{2}\right)$. Let us note $x_{k}$, for $k$ in $\mathbb{Z}^{2}$, the point of coordinates $\left(k_{1} h_{1}, k_{2} h_{2}\right)$ and $Q=$ $\left\{k \in \mathbb{Z}^{2}\right.$ such that $\left.x_{k} \in \bar{\Omega}\right\}$. We call "pixel" a point $x_{k}$ in $\bar{\Omega}$. From each "generic SFS" approximation scheme (the implicit one and the semi-implicit one), we can associate an algorithm that computes for all $k \in Q$ a sequence of approximations $U_{k}^{n}$ of $u^{\rho}\left(x_{k}\right)$ :

\section{Algorithm}

1. Initialisation $(n=0): \quad \forall k \in Q, \quad U_{k}^{0}=u_{0}\left(x_{k}\right)$. Choose the first pixel $x_{k}$.

2. Modification of $U^{n}($ step $n+1)$ : we set

$$
U_{k}^{n+1}=\max \left\{t \mid S\left(\rho, x_{k}, t, U^{n}\right)=0\right\}
$$


and we do not change the other values: $\forall l \neq k$, $U_{l}^{n+1}=U_{l}^{n}$.

3. Choose the next pixel $x_{k}$ in such a way that all pixels are regularly visited and go back to 2 (since $\bar{\Omega}$ is bounded, the number of pixels is finite).

In this iterative algorithm, the initial surface $u_{0}$ is a subsolution or a supersolution of the considered scheme. We have detailed in section 4.3 the subsolutions of the implicit approximation scheme (note that the semi-implicit algorithm starting from a subsolution is really not efficient). In practice, when we start from a supersolution, we do not actually compute it. In effect a large constant function $u_{0}$ with the appropriate boundary conditions is sufficient. Let us also remark that the speed of convergence strongly depends on the initial surface $u_{0}$. Experimentally we found that the speed of convergence is much higher when we start from a supersolution; a quantitative comparison is found in section 6 . The convergence speed also depends on the particular path used to traverse the set of pixels. In our implementation, we have chosen the strategy which consists in following the path indicated in figure 6. Similar alternating raster scans were yet proposed by Danielsson [15] in the 80's and were used by Rouy and Tourin [55] and Dupuis and Oliensis [16, 17]. Also, even if this strategy is not optimal in the computational complexity sense (see section 6.4 for more details), in practice it is very effective (at least on all the real images we have tested) and it is extremely simple to implement.

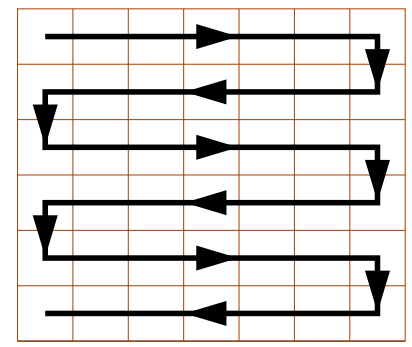

Figure 6: Alternating raster scans strategy $[15,16,17,55]$.

We have designed two new "generic SFS" algorithms with which we can compute numerical solutions of each formulation of the SFS problem. Moreover, in [51] we have proved that, when $u_{0}$ is a subsolution or a supersolution, the numerical solutions (computed with either one of the two algorithms) converge toward the solutions of the approximation schemes. Note that this holds for all intensity images $I$ : No regularity hypotheses are required. In particular, the convergence holds for discontinuous images and images containing black shadows (i.e. zones with 0 intensity). We have implemented the algorithms associated with the implicit "generic SFS" approximation scheme and with the semi-implicit "generic SFS" approximation scheme. The resulting code applies to all the SFS Hamiltonians described in section 3.3.1. As mentioned before, the interest of the "generic" formulation of the SFS problems lies in the fact that the same code can be used to solve a variety of different problems, e.g. the "perspective SFS" and the "orthographic SFS" problems. On the other hand, because of this generality, we may lose optimality for a particular case.

Finally, let us mention that the algorithm proposed by Rouy and Tourin in [55] is the implicit algorithm applied to the Eikonal Hamiltonian $H_{\text {Eiko. }}^{\text {orth }}$ The controlbased algorithm proposed by Dupuis and Oliensis in [17] is the semi-implicit algorithm applied to the Hamiltonian $H_{D / O}^{\text {orth }}$. The algorithms we have proposed in $[53,52]$ are the implicit algorithm applied to $H_{R / T}^{\text {orth }}$ and $H_{P / F}^{\text {pers }}$, respectively. Therefore, from an algorithmic point of view, our work can be interpreted as a generalization and a unification of the work of Rouy and Tourin [55], the work of Prados and Faugeras [53, 52] and the work of Dupuis and Oliensis [17].

As a final remark we note that in $[55,53,52]$ the authors use subsolutions as initial conditions, whereas in [17], they use supersolutions.

\section{Experimental results}

We have implemented the algorithms associated with the implicit and (optimal) semi-implicit approximation schemes for the "generic SFS" Hamiltonian $H_{g}$. In the following subsections, we compare the results obtained with our algorithms. This comparison is based on the speed of convergence and the reconstruction error. We start with the algorithms associated with the orthographic SFS problem. In this context, we emphasize the comparison of the implicit and semi-implicit algorithms, and the 
influence of the initial surface $u_{0}$ on the speed of convergence. We have tested our algorithms with synthetic images generated by shapes with several degrees of regularity e.g. $C^{\infty}$ (a paraboloid, a sinusoid and a smoothed vase, see figures $7,8,14$ ), or $C^{0}$ (a pyramid, see figures 9, 15 ), to demonstrate the ability of our method to work with smooth and nonsmooth objects. We have also tested our algorithms on more complicated images; for example, the classical Mozart's face ${ }^{28}$; see figures 1016 and 17. Next, we deal with the perspective SFS algorithms. In particular we compare the results obtained by the orthographic SFS algorithms and the perspective SFS algorithms for synthetic perspective images.

In all the examples, the parameters are $n$, the number of iterations, $\varepsilon_{1}, \varepsilon_{2}$ and $\varepsilon_{\infty}$ the mean absolute errors between the reference and reconstructed surfaces measured according to the $L_{1}, L_{2}$ and $L_{\infty}$ norms, respectively, $\theta$ the angle of the direction of illumination with the $z$-axis. We note $\mathbf{L}=(1, \gamma)$ the light vector and $f$ the focal length

According to the theory we have developed in this article there exist in general several viscosity solutions. In order to have uniqueness we need to impose Dirichlet boundary conditions on $\partial \Omega^{\prime}=\partial \Omega \cup\{x \mid I(x)=1\}$ (see section 3.5). This means that we must provide the "height" of the solution at the boundary of the image and at all singular points (i.e. the pixels $x_{i j}$ such that $\left.I\left(x_{i j}\right)=1\right)$. This is one of the reasons why we present our results on synthetic images. Note that in [53], we have shown a reconstruction of a Halloween mask from a real image.

\subsection{Experimental results in the case of "or- thographic SFS"}

We tested the orthographic SFS algorithms with synthetic images generated by an orthographic projection. In all cases, we show the original object, the input image and the reconstructed surface. First we show that the accuracy of the implicit algorithm is approximately the same as that of the semi-implicit algorithm. This confirms the prediction of the theory that an implicit scheme and its associated semi-implicit scheme have the same solutions, and

\footnotetext{
${ }^{28}$ The synthetic surfaces of the vase and of Mozart's face are associated to the paper by Zhang et al. [64] (Computer Vision Lab. of the university of Central Florida).
}

that the computed numerical approximations converge towards the solution of these schemes. Figures 7 and 8 show the reconstructions of smooth surfaces obtained by the implicit algorithm (associated with the Hamiltonian $\left.H_{R / T}^{o r t h}\right)$ and by the semi-implicit algorithm, starting from a subsolution and from a supersolution.

Since, in practice, the combination (semi-implicit algorithm, subsolution) is not really effective, we only show the results obtained with the other three combinations. As shown in the figures we recover in these three cases almost exactly the same surface. On the other hand the numbers of iterations required for converging are very different. Globally, the number of iterations required for converging with a semi-implicit algorithm is much larger than with an implicit algorithm. For example, when $u_{0}$ is a supersolution, approximately 100 iterations are required for obtaining the sinusoidal surface with the semi-implicit algorithm (figure 8-d), when only 20 iterations are sufficient with the implicit algorithm (figure 8-e). Furthermore, the number of iterations required when the approximation sequence starts from a subsolution is much larger than when it starts from a supersolution. For the example of the sinusoidal surface displayed in figure 8 , the implicit algorithm requires approximately 600 iterations for converging when $u_{0}$ is a subsolution; when only $\simeq 20$ iterations are required when $u_{0}$ is a supersolution.

Figure 10 shows the speed of convergence of the two algorithms for two different initial conditions, i.e. a subsolution (except for the semi-implicit scheme, as mentioned above) and a supersolution. Clearly, as shown in tables 1-3, the combination (implicit, supersolution) is the best.

To demonstrate the ability of our method to deal with nonsmooth objects, we have tested our algorithms with a pyramidal surface, see figure 9. The previous remarks about accuracy and speed of convergence still hold for nonsmooth surfaces.

We also show the stability of our method with respect to two types of errors. The first type is image intensity errors due to noise. Uniformly distributed white noise has been added to all pixels of the input images and the corresponding reconstructed surfaces are shown, see figure 11 for the sinusoidal surface and 12 for the pyramidal surface. The Signal to Noise Ratio (SNR) is equal to 3.2 in figure 11 and to 2.7 in figure 12. As seen from these figures, our algorithms are very robust to intensity noise, as also observed in $[55,17]$. The second type of error is due to an 
incorrect estimation of the direction of the illumination $\mathbf{L}$. Starting with the sinusoidal object of figure 8 , we show in figure $13-\mathrm{a}$. that an error of roughly $9^{\circ}$ on the parameter $\mathbf{L}$ does not affect much the reconstructed surface whereas for a larger error of $15^{\circ}$, the result is more distorted. We continue with the pyramidal shape of figure 9; figure 13b) shows a similar trend: a small error of approximately $5^{\circ}$ already affects the results and later we introduce a large error of $\left(9^{\circ}\right)$. Our algorithms seem to be fairly robust to small inaccuracies in the estimation of the direction of the light source L (Fig.13-a-1 and Fig.13-b-1). Nevertheless, when the error grows larger, the results degrade rapidly because some undesirable edges are created. Finally, let us remind that the robustness we have demonstrated experimentally here confirms the theoretical stability results proved in section 3.6.

\subsection{Experimental results in the case of "per- spective SFS',}

We have tested the perspective algorithms with synthetic images generated by using a perspective projection. The previous remarks about the speed of convergence of the orthographic SFS algorithms still hold for the perspective SFS algorithms. In the following results, the solutions are computed with the implicit algorithm associated with the Hamiltonian $H_{P / F}^{\text {pers }}$ starting from a subsolution (figures 14, 15 and 16) or from a supersolution (figures 17 and 18). In figures 14, 15 and 16, we show the original object, the input image, the surface reconstructed by our "perspective algorithm" and the surface reconstructed by the "orthographic algorithm". We denote $r$ the ratio of the focal length and object distance (the object distance is the mean distance of the points on the surface to the optical center). Notice that, as soon as the ratio $r$ grows larger than 1.5, the "orthographic algorithm" produces important errors whereas the quality of the results obtained by the "perspective algorithm" are very good (see figures 14, 15 and 16). This shows the importance of taking into account the perspective distortion in the SFS problem.

As for the orthographic algorithms, we demonstrate the stability of the perspective SFS algorithms with respect to various types of errors. The first type is due to noise in the image intensity (see figure $17, \mathrm{SNR} \simeq 3.7$ ). The second type of error is due to an incorrect estimation of the

\begin{tabular}{||l||c|c||}
\hline iteration & $\varepsilon_{2}$ error & $\varepsilon_{\infty}$ error \\
\hline \hline 30 & 0.0379 & 0.1123 \\
\hline 60 & 0.0244 & 0.0664 \\
\hline 90 & 0.0178 & 0.0500 \\
\hline 120 & 0.0128 & 0.0391 \\
\hline 150 & 0.0086 & 0.0337 \\
\hline 200 & 0.0032 & 0.0336 \\
\hline
\end{tabular}

Table 1: Errors associated to figure 10 for the implicit algorithm starting from a subsolution.

\begin{tabular}{||l||c|c||}
\hline iteration & $\varepsilon_{2}$ error & $\varepsilon_{\infty}$ error \\
\hline \hline 8 & 0.0358 & 0.0882 \\
\hline 16 & 0.0157 & 0.0562 \\
\hline 24 & 0.0086 & 0.0390 \\
\hline 32 & 0.0058 & 0.0335 \\
\hline 40 & 0.0042 & 0.0335 \\
\hline 48 & 0.0033 & 0.0335 \\
\hline
\end{tabular}

Table 2: Errors associated to figure 10 for the semiimplicit algorithm starting from a supersolution.

direction of illumination $\mathbf{L}$ (see figure 18-a). The third type of error is due to an incorrect estimation of the focal length (see figure 18-b). As seen from these figures, the algorithms are quite robust to intensity noise; they are also robust to small inaccuracies in the light and focal parameters. But large errors on these parameters create some spurious edges.

\begin{tabular}{||l||c|c||}
\hline iteration & $\varepsilon_{2}$ error & $\varepsilon_{\infty}$ error \\
\hline \hline 4 & 0.0046 & 0.0432 \\
\hline 8 & 0.0034 & 0.0333 \\
\hline 12 & 0.0032 & 0.0336 \\
\hline 16 & 0.0032 & 0.0336 \\
\hline 20 & 0.0032 & 0.0336 \\
\hline 24 & 0.0032 & 0.0336 \\
\hline 28 & 0.0032 & 0.0336 \\
\hline
\end{tabular}

Table 3: Errors associated to figure 10 for the implicit algorithm starting from a supersolution. 


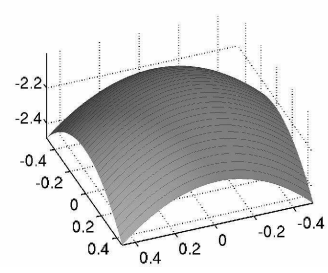

a)

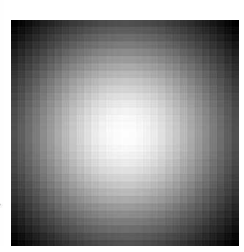

b)

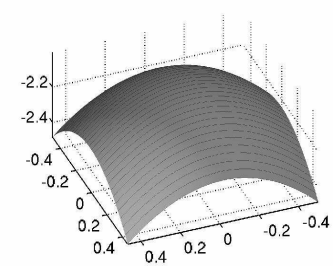

c)

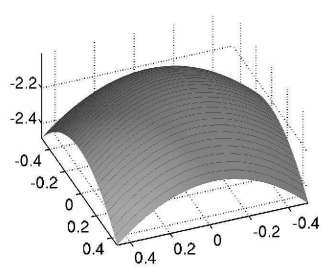

d)

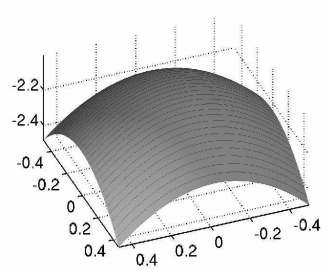

e)

Figure 7: Results for a synthetic image of a paraboloidal surface sampled on a $32 \times 32$ grid with $1=(0,0)\left(\theta \simeq 0^{\circ}\right)$ : a) original surface (groundtruth), b) original image, c) surface reconstructed from b) with the implicit algorithm starting from a subsolution: $n=18, \varepsilon_{1}=0.0015, \varepsilon_{2}=0.0018, \varepsilon_{\infty}=0.0021$; d) surface reconstructed from b) with the semi-implicit algorithm starting from a supersolution: $n=15, \varepsilon_{1}=0.0014, \varepsilon_{2}=0.0016, \varepsilon_{\infty}=0.0020$; e) surface reconstructed from $\mathrm{b}$ ) with the implicit algorithm starting from a supersolution: $n=5, \varepsilon_{1}=0.0015, \varepsilon_{2}=0.0018$, $\varepsilon_{\infty}=0.0020$;

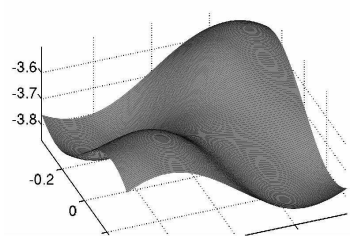

a)

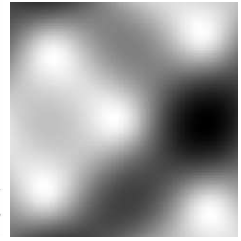

b)

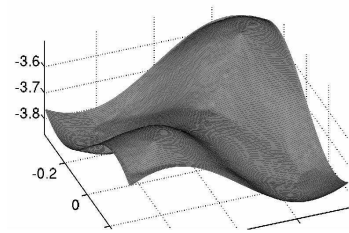

c)

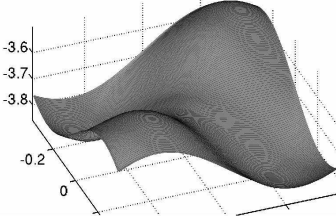

d)

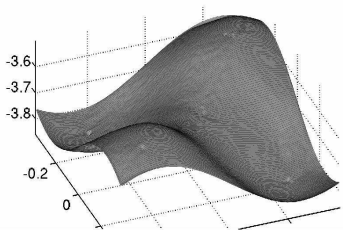

e)

Figure 8: Results for a synthetic image of a sinusoidal surface sampled on a $200 \times 200$ grid with $\mathbf{l}=(0.1,0.3)$ $\left(\theta \simeq 18.5^{\circ}\right)$ : a) original surface, b) original image, c) surface reconstructed from b) with the implicit algorithm starting from a subsolution: $n \simeq 700, \varepsilon_{1}=0.003902, \varepsilon_{2}=0.005762, \varepsilon_{\infty}=0.00740$; d) surface reconstructed from b) with the semi-implicit algorithm starting from a supersolution: $n \simeq 120, \varepsilon_{1}=0.003900, \varepsilon_{2}=0.005762$, $\varepsilon_{\infty}=0.00747$; e) surface reconstructed from b) with the implicit algorithm starting from a supersolution: $n \simeq 25$, $\varepsilon_{1}=0.003905, \varepsilon_{2}=0.005768, \varepsilon_{\infty}=0.00747$;

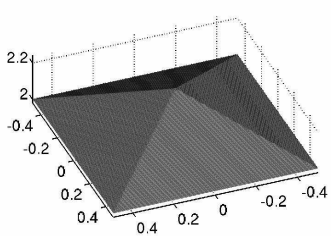

a)

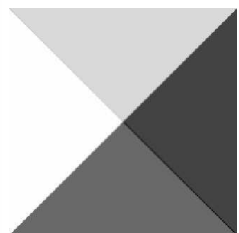

b)

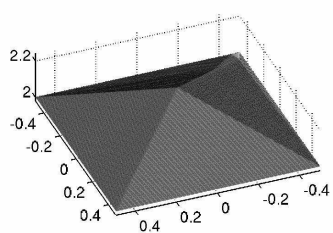

c)

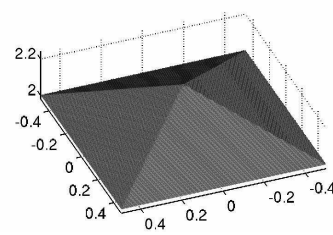

d)

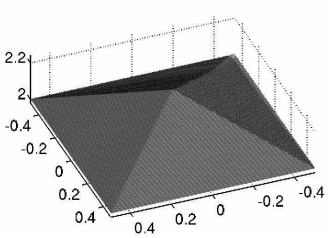

e)

Figure 9: Results for a synthetic image of a pyramidal surface sampled on a grid of size $200 \times 200$ with $\mathbf{l}=(0.5,0.3)$ $\left(\theta \simeq 35.6^{\circ}\right)$ : a) original surface, b) original image, c) surface reconstructed from b) with the implicit algorithm starting from a subsolution: $n \simeq 1000, \varepsilon_{1}=8.461 e-05, \varepsilon_{2}=1.6116 e-04, \varepsilon_{\infty}=9.40 e-04$; d) surface reconstructed from b) with the semi-implicit algorithm starting from a supersolution: $n \simeq 110, \varepsilon_{1}=8.461 e-05, \varepsilon_{2}=1.6116 e-04$, $\varepsilon_{\infty}=9.40 e-04$; e) surface reconstructed from b) with the implicit algorithm starting from a supersolution: $n \simeq 50$, $\varepsilon_{1}=8.461 e-05, \varepsilon_{2}=1.6116 e-04, \varepsilon_{\infty}=9.40 e-04$; 

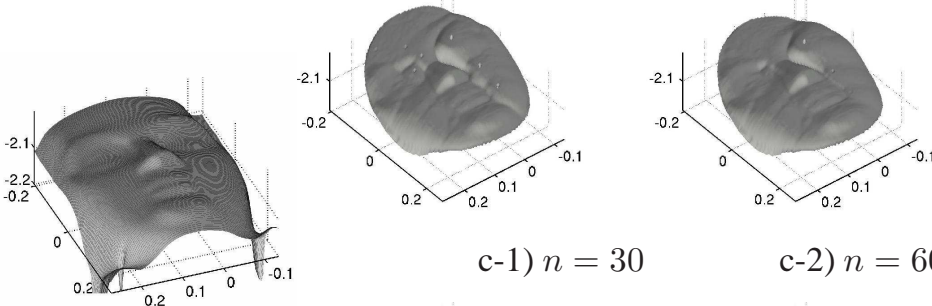

c-1) $n=30$

c-2) $n=60$

a)

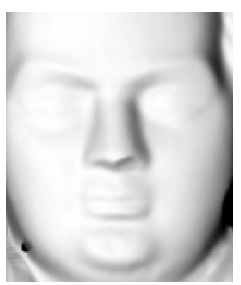

b)
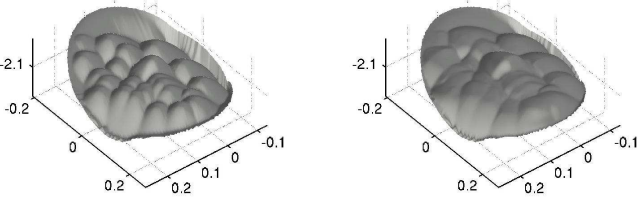

d-1) $n=8$

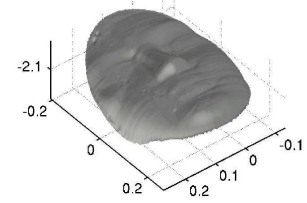

e-1) $n=4$

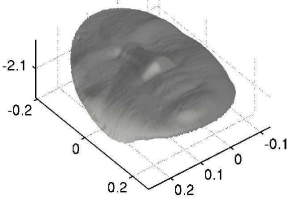

e-2) $n=8$

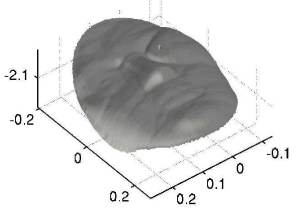

c-3) $n=120$

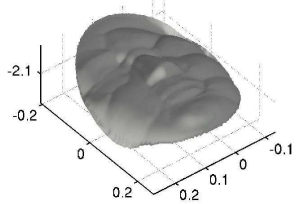

d-3) $n=24$

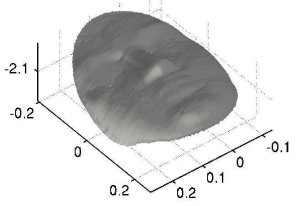

e-3) $n=12$

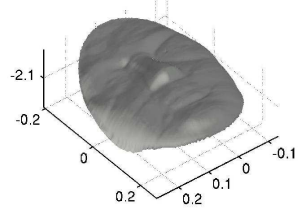

c-4) $n=150$

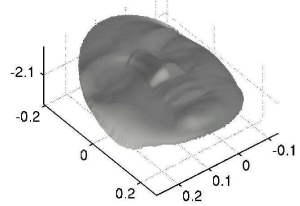

d-4) $n=40$

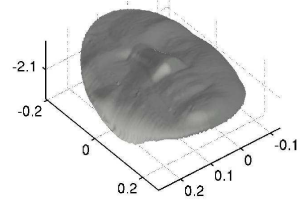

e-4) $n=16$

Figure 10: Experimental results obtained with the implicit/semi-implicit algorithms starting from a subsolution/supersolution, for a synthetic image representing Mozart's face: a) Original surface of size $\simeq 150 \times 150$, b) synthetic image generated from the original surface a) with $\mathbf{l}=(0.2,0.1)\left(\theta \simeq 13^{\circ}\right)$; c-1) to c-4) surface $U^{n}$ recontructed with the implicit algorithm starting from a subsolution at the $n^{\text {th }}$ iteration for $n=30, n=60, n=120$ and $n=150$, respectively; d-1) to d-4) surface $U^{n}$ recontructed with the semi-implicit algorithm starting from a supersolution at the $n^{\text {th }}$ iteration for $n=8, n=16, n=24$ and $n=40$, respectively; e- 1 ) to e-4) surface $U^{n}$ recontructed with the implicit algorithm starting from a supersolution at the $n^{\text {th }}$ iteration for $n=4, n=8, n=12$ and $n=16$, respectively. The corresponding reconstruction errors are shown in tables 1-3.

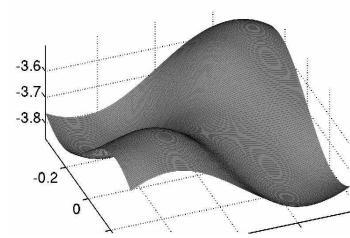

a)

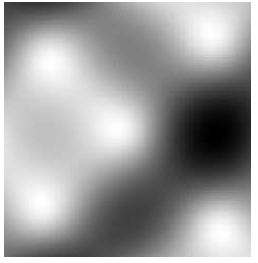

b)

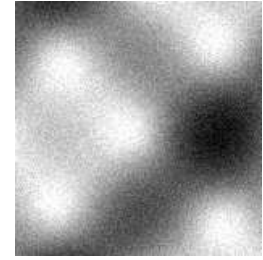

c)

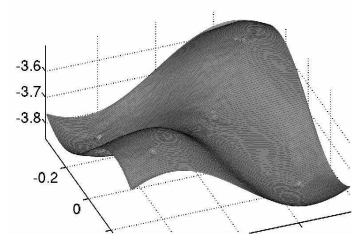

d)

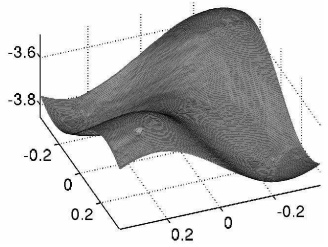

e)

Figure 11: Results for a noisy image of a sinusoidal surface sampled on a $200 \times 200$ grid with $\mathbf{l}=(0.1,0.3)(\theta=$ $18.5^{\circ}$ ). a) Original surface, b) original image, c) roisy image; d) reconstructed surface from b): $n \simeq 25, \varepsilon_{1}=$ $0.003905, \varepsilon_{2}=0.005768, \varepsilon_{\infty}=0.00747$; e) reconstructed surface from c): $n \simeq 30, \varepsilon_{1}=0.003905, \varepsilon_{2}=0.005766$, $\varepsilon_{\infty}=0.00748$ 


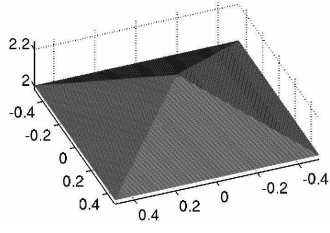

a)

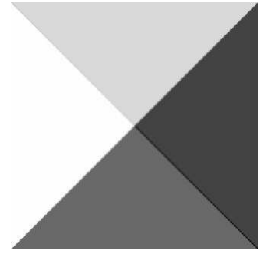

b)

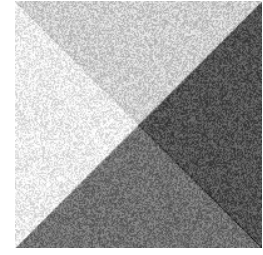

c)

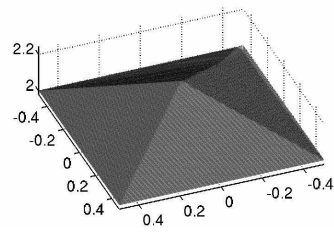

d)

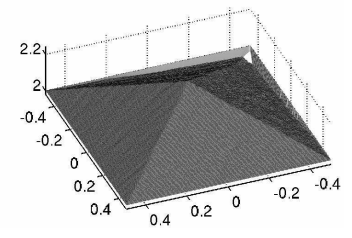

e)

Figure 12: Results for a noisy image of a pyramidal surface sampled on a $200 \times 200$ grid with $\mathbf{1}=(0.5,0.3)$ $\left(\theta=35.6^{\circ}\right)$. a) Original surface, b) original image, c) noisy image; d) surface reconstructed from b): $n \simeq 50$, $\varepsilon_{1}=8.461 e-05, \varepsilon_{2}=1.6116 e-04, \varepsilon_{\infty}=9.4000 e-04 ;$ e) surface reconstructed from c): $n \simeq 50, \varepsilon_{1}=0.00467$, $\varepsilon_{2}=0.00916, \varepsilon_{\infty}=0.044$.

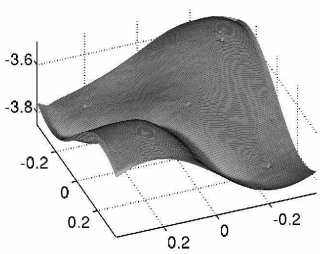

a-1)

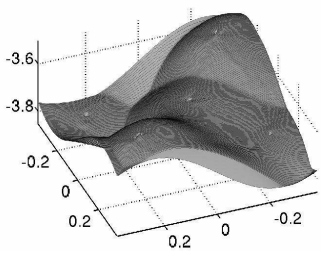

a-2)

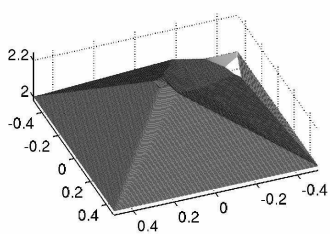

b-1)

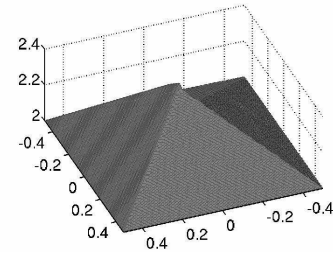

b-2)

Figure 13: Sinusoidal (respectively pyramidal) surface of figure 8 (respectively of figure 9) reconstructed from the image Fig.8-b) (respectively from Fig.9-b) with an error on the light parameter L. The light parameter used for obtaining the image 8 -b) was $\mathbf{l}=(0.1,0.3)$ (respectively $\mathbf{l}=(0.5,0.3)$ ). a-1) sinusoidal surface reconstructed with $1=(0.0,0.3)\left(\varepsilon_{\theta} \simeq 9.3^{\circ}\right): n \simeq 40, \varepsilon_{1}=0.0171, \varepsilon_{2}=0.0314, \varepsilon_{\infty}=0.0729$; a-2) sinusoidal surface reconstructed with $\mathrm{l}=(0.3,0.2)\left(\varepsilon_{\theta} \simeq 15.4^{\circ}\right): n \simeq 35, \varepsilon_{1}=0.0394, \varepsilon_{2}=0.0684, \varepsilon_{\infty}=0.142$. b-1) pyramidal surface reconstructed with $1=(0.3,0.2) \quad\left(\varepsilon_{\theta} \simeq 5.3^{\circ}\right): n \simeq 40, \varepsilon_{1}=0.0407, \varepsilon_{2}=0.0556, \varepsilon_{\infty}=0.177 ; \quad$ b-2) pyramidal surface reconstructed with $\mathbf{l}=(0.4,0.4) \quad\left(\varepsilon_{\theta} \simeq 8.8^{\circ}\right): n \simeq 40, \varepsilon_{1}=0.0251, \varepsilon_{2}=0.0334, \varepsilon_{\infty}=0.103$.

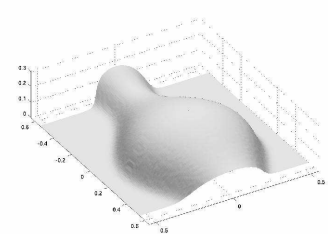

a)

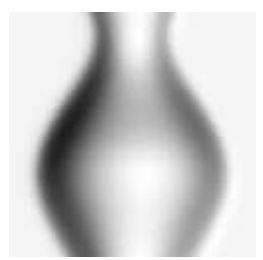

b)

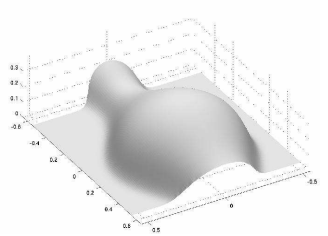

c)

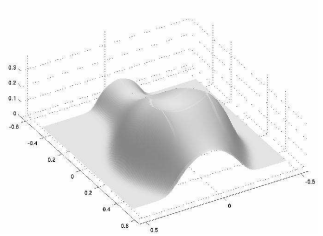

d)

Figure 14: "Perspective SFS" results for an image of a smooth surface (computed by the implicit algorithm starting from a subsolution): a) original surface, b) original image $(l=(0.2,0.2), r=2.5$, size $=128 \times 128)$, c) surface reconstructed from $b$ ) by the "perspective algorithm": $n \simeq 1000, \varepsilon_{1}=0.0041, \varepsilon_{2}=0.0048, \varepsilon_{\infty}=0.00814 ;$ d) surface reconstructed from b) by the "orthographic algorithm": $n \simeq 1000, \varepsilon_{1}=0.0201, \varepsilon_{2}=0.031, \varepsilon_{\infty}=0.035$; 


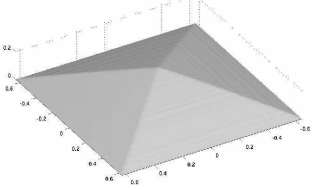

a)

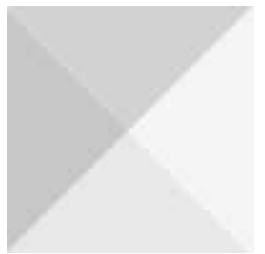

b)

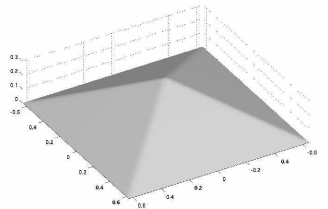

c)

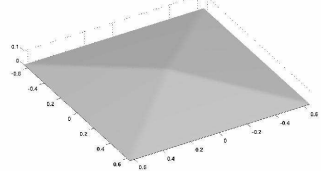

d)

Figure 15: "Perspective SFS" results for an image of a pyramidal surface (computed by the implicit algorithm starting from a subsolution): a) original surface, $b)$ original image $(l=(0.2,0.2), r=2.1$, size $=100 \times 100), \quad$ c) surface reconstructed from b) by the "perspective algorithm": $n \simeq 76, \varepsilon_{1} \simeq 0.00015, \varepsilon_{2} \simeq 0.0009, \varepsilon_{\infty} \simeq 0.00110 ;$ d) surface reconstructed from $b$ ) by the "orthographic algorithm": $n=\simeq 83, \varepsilon_{1}=0.063, \varepsilon_{2}=0.130, \varepsilon_{\infty}=0.135$;

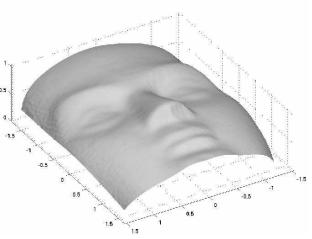

a)

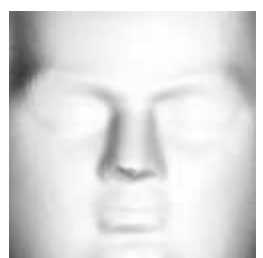

b)

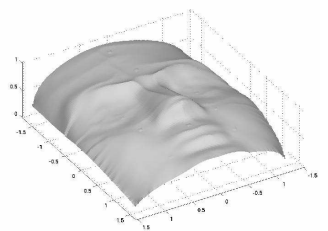

c)

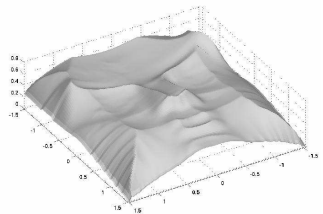

d)

Figure 16: "Perspective SFS" results for an image of Mozart's face (computed by the implicit algorithm starting from a subsolution): a) original surface, b) original image $(l=(0.1,0.1), r \approx 1.6$, size $=128 \times 128)$, c) surface reconstructed from b) by the "perspective algorithm": $n \simeq 4000, \varepsilon_{1} \simeq 0.00255, \varepsilon_{2} \simeq 0.004 .14976, \varepsilon_{\infty} \simeq 0.012$; d) surface reconstructed from $b$ ) by the "orthographic algorithm": $n \simeq 5000, \varepsilon_{1}=0.0495, \varepsilon_{2}=0.1187, \varepsilon_{\infty}=0.20$;

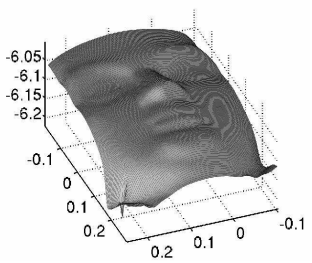

a)

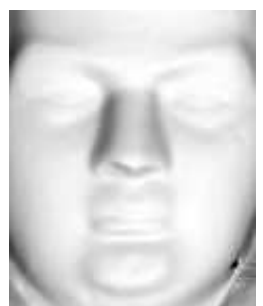

b)

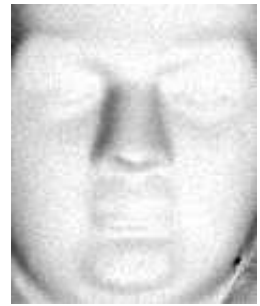

c)

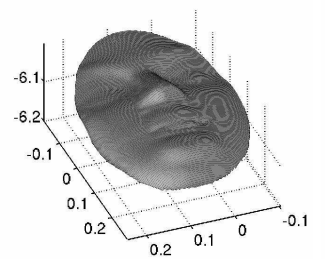

d)

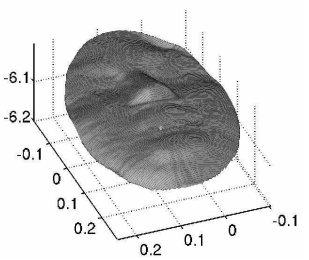

e)

Figure 17: "Perspective SFS" results for an image of Mozart's face corrupted by a uniformly distributed noise (Size of the grid $\simeq 200 \times 200$; light parameter: $1=(0.1,0.3), \theta=18.4^{\circ}$, focal length: $\left.f=4\right)$. a) Original surface, b) original image, c) noisy image; d) surface reconstructed from b): $n \simeq 5, \varepsilon_{1}=0.00197, \varepsilon_{2}=0.00338, \varepsilon_{\infty}=0.00721$; e) surface reconstructed from c): $n \simeq 7, \varepsilon_{1}=0.00247, \varepsilon_{2}=0.00450, \varepsilon_{\infty}=0.0116$. 


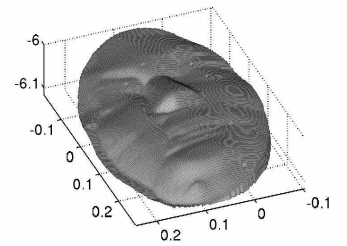

a-1)

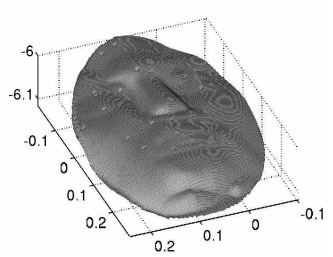

$\mathrm{a}-2)$

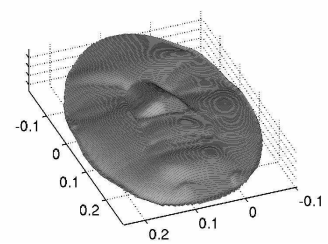

b-1)

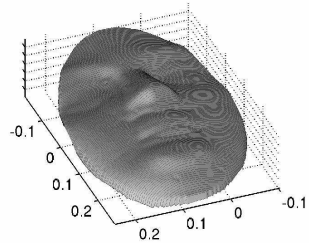

b-2)

Figure 18: "Perspective SFS" results for Mozart's face of figure 17-a) reconstructed with an error on the light parameter $\mathbf{l}$ (respectively on the focal length parameter $f$ ): The light parameter $\mathbf{l}$ used for synthesizing image $17-\mathrm{b}$ ) is $\mathbf{l}=$ $(0.1,0.3)$ and the focal length parameter $f$ is equal to 5 . a-1) Result obtained with the corrupted parameter $\mathbf{l}=$ $(0.3,0.3) ; n \leq 10\left(\varepsilon_{1}=0.0131, \varepsilon_{2}=0.0244, \varepsilon_{\infty}=0.0466\right)$. a-2) Result obtained with the corrupted parameter $\mathbf{l}=(0.1,0.5) ; n \leq 10\left(\varepsilon_{1}=0.0226, \varepsilon_{2}=0.0396, \varepsilon_{\infty}=0.0547\right)$. b-1) Result obtained with the corrupted focal length $f=4 ; n \simeq 6$. b-2) Result obtained with the corrupted focal length $f=6 ; n \leq 6$.

\subsection{Cases of degeneracy}

By considering for example the Hamiltonian $H_{R / T}^{\text {orth }}$, one can verify that, in a neighbourhood of a singular point, the equation in $t$ associated to the implicit SFS scheme

$$
S\left(\rho, x_{i j}, t, U\right)=0
$$

is almost degenerate, i.e. may not have any solutions (see [51] for more details). This is true of all schemes arising form the generic Hamiltonian $H_{g}$ and is due to the fact that we are computing the zero-crossings of parabola-like curve; when the intensity is equal to 1 this curve is almost tangent to the horizontal axis and the roots become unstable. The implication of this observation is that if we are not careful when solving equation (36), it is possible that numerically, we do not obtain any solutions $(36)^{29}$ (even if theorically there exists a solution).

In order to combat this problem we have used three strategies. First, instead of solving the equation (36), we can compute the value $t$ which minimizes $S\left(\rho, x_{i j}, t, U\right)$. Second, we can change slightly the values of the intensity image, and introduce the image $I_{\varepsilon}$ such that $I_{\varepsilon}(x)=I(x)$ if $I(x)<1-\varepsilon$ and $I_{\varepsilon}(x)=1-\varepsilon$ otherwise (for some small $\varepsilon>0$ ). The idea consisting in using $I_{\varepsilon}$ instead of $I$ has been already used by Horn [25], Kimmel and Bruckstein [35] and Falcone et al. [8]. Third, we can update the values of $U$ with the semi-implicit algorithm when we

\footnotetext{
${ }^{29}$ Let us note that, in practice, we have been rarely confronted to this
} situation. have not found a solution during the updating with the implicit algorithm.

Let us emphasize the fact that, even when we do not use any of these three strategies (for example when we do not find a solution of (36) we can simply not update the current value), these rare and undesirable events do not affect the overall quality of the reconstruction at the other points. This shows again the very nice stability properties of our algorithms.

\subsection{What about other approaches?}

The task of comparing our algorithms with already published ones is way beyond the scope of this paper. As shown in figures 10, 17 and 18 the ("generic SFS") implicit algorithm (starting from a supersolution) returns, in most of our examples, quite good results after only four iterations (very often, the solutions returned after only two or three iterations are visually quite good). This should not come as a surprise since, as pointed out at the end of section 5, our implicit algorithms generalize that of Rouy and Tourin [55] and our semi-implicit algorithms generalize that of Dupuis and Oliensis [17]. In the literature these algorithms are often acknowledged as being one of the most efficient and accurate of the SFS literature.

As pointed out in section 5 our algorithms are iterative and their convergence speed strongly depends on the chosen paths ordering the updates. In our implementation we have used alternating raster scans $[15,17]$. These scans are not optimal (because they are arbitrary with re- 
spect to the characteristics of the solution) and the method can be improved by tracing directly the characteristics, as been done by Sethian et al. [57, 36] (this will be the concern of a furthcoming paper). This techniques would allow to decrease the computational complexity by one order of magnitude $\left(O(n)\right.$ instead of $\left.O\left(n^{2}\right)\right)$. Let us remind that our generic implicit scheme is an extension of the scheme used by Sethian in the Fast Marching Method for the eikonal equation [57].

\section{Pushing things to the limit: SFS with discontinuous images and black shadows}

Among the difficulties encountered when attempting to solve the SFS problem, the intensity discontinuities such as those caused by black shadows are among the most difficult to deal with. Despite the fact that the notion of viscosity solutions provides a natural framework for dealing with non smooth surfaces ${ }^{30}$ (with edges) this theory does not yet apply to discontinuous images ${ }^{31}$ (and hence to black shadows). Technically, when the Hamiltonian is discontinuous with respect to the space variable $x$ (which is the case in SFS when the intensity image is discontinuous), the main difficulty is the loss of uniqueness of the viscosity solution. Note that, in the particular case of the black shadows, this difficulty is increased by the loss of coercivity of the Hamiltonian.

In order to deal with black shadows, Lions et al. [42] do not "recover" surfaces in the areas of 0 intensity and pose the problem in terms of boundary conditions. This is not necessary since, as noted in [23], in the black shadows areas the surface formed by the rays of light grazing the solution surface, verify the irradiance equation, see figure 19. Thus, for recovering a solution, we do not need, as in [42], to separate the "shading areas" and the "shadow areas" and in general ${ }^{32}$ our generic algorithm graciously computes approximations of the exact solutions in shading areas and the grazing rays of light in the black shad-

\footnotetext{
${ }^{30}$ Viscosity solutions are weak (i.e. non differentiable) solutions.

${ }^{31}$ Leaning on some recent work $[47,32,58,10]$ on the Eikonal equation we are working on the removing of this limitation.

${ }^{32}$ If we assume that the singular points and the boundary of the image are not covered by the shadows.
}

ows areas (as does the algorithm proposed by Falcone [23] for the "orthographic SFS"), see figures 19 and 20.

Finally the stability of our approximation schemes and the convergence of the numerical solutions computed by our algorithms hold even when the image contains discontinuities and black shadows, even though the theory of viscosity solutions does not yet apply to this case, see the remark at the end of section 4.3. As an illustration of this, the pyramid example displayed in figures 9, 12 and 15 shows the ability of our numerical algorithms to deal with discontinuous images while figure 20 shows their ability to deal with black shadows.

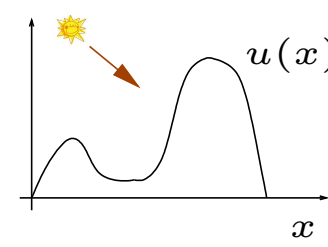

a)

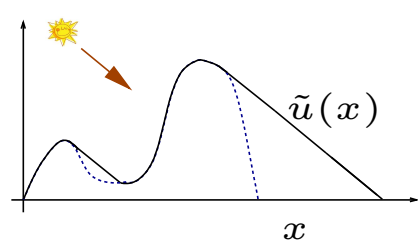

b)
Figure 19: a) Original surface $u$; b) Solution computed by our and Falcone's algorithms [23].

\section{Conclusion}

We have presented a complete mathematical and algorithmic study of the "orthographic" and "perspective SFS" problems. In detail: 1) We have proposed new formulations of the SFS problem by modeling the camera as a pinhole (performing a perspective rather than orthographic projection). The scene can be illuminated by a single point light source located at infinity or at the optical center of the camera. This extends the SFS methods to more realistic image acquisition models. These formulations lead to new PDEs which allow to develop a complete mathematical study of the problem. 2) By using the theory of viscosity solutions, we have proved the existence and characterized the solutions of the "orthographic" and "perspective SFS" problems. In particular, this allows us to choose a particular solution of interest before starting to produce numerical results. 3) By introducing a "generic" Hamiltonian, we have unified the "orthographic" and "perspective SFS" problems, and simplified 


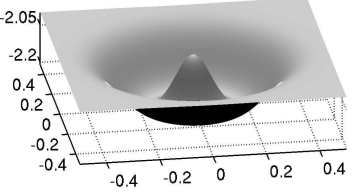

a)

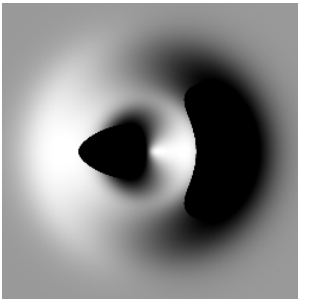

b)

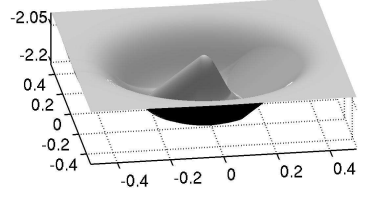

c)

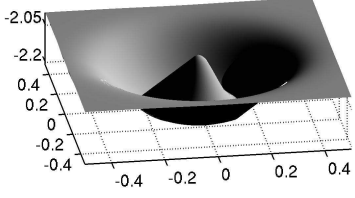

d)

Figure 20: Example of a reconstruction from an image with black shadows: the case of a "Mexican hat" (Size of the image $\simeq 1000 \times 1000$, result computed by the implicit algorithm starting from a supersolution: $n<90$.) a) Original surface (the direction of the visualisation light is $(0,0,1)$ ) and is different from $\mathbf{L}$; b) Synthetic image computed from the surface a) with $\mathbf{L}=(0.8,0.0,0.6)$ (the angle between the light direction $\mathbf{L}$ and the camera axis is around $53^{\circ}$ ); c) Solution recovered by our algorithm from the image b) (the direction of the visualisation light is $(0,0,1))$; d) Surface c) illuminated by a light of direction $(0.8,0.0,0.6)$.

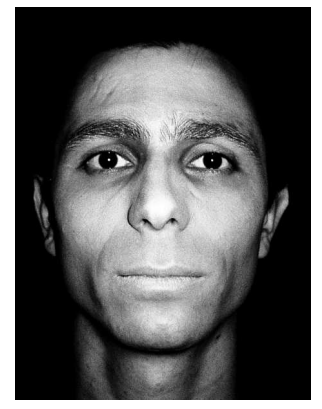

a)

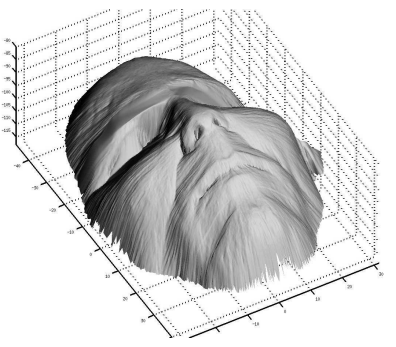

b)

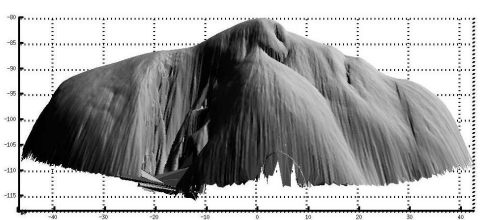

c)

Figure 21: a) Real face image [size $\simeq 450 \times 600$ ]; b-c) surface recovered from a) by our generic algorithm with the perspective model with the light source located at the optical center with a one-point Dirichlet boundary condition. 
the formalism. We have designed two "generic" approximation schemes which approximate the "generic SFS" equation. From these approximation schemes we have obtained two "generic SFS" algorithms. Each "generic SFS" algorithm can be used to solve numerically the various formulations of the SFS problem. Moreover, we have proved the convergence of the numerical solutions computed by our algorithms toward the viscosity solutions of the considered SFS problem. 4) Our algorithms are robust to pixel noise and to the errors made on the parameters. 5) They can deal with discontinuous images and images containing black shadows. We have proved the stability of our SFS approximation schemes and the convergence of our SFS algorithms with such images. We are extending our approach to be able to remove the requirement for the knowledge of the boundary data (Dirichlet conditions at the singular points and on the boundary of the image) and for recovering non Lambertian surfaces. To give a flavor of what could be achievable figure 21 shows the surface recovered by our generic algorithm (after 5 iterations) with the perspective model with a point light source at the optical center. In this example, we have sent to infinity all the points on the boundary of the image and all the singular points except the one on the nose at which we have specified a "reasonable" depth.

\section{References}

[1] M. Bardi and I. Capuzzo-Dolcetta. Optimal control and viscosity solutions of Hamilton-Jacobi-Bellman equations. Birkhauser, 1997.

[2] G. Barles. Solutions de Viscosité des Equations de Hamilton-Jacobi. Springer-Verlag, 1994.

[3] G. Barles and P.E. Souganidis. Convergence of approximation schemes for fully nonlinear second order equations. Asymptotic Analysis, 4:271-283, 1991.

[4] M.J. Brooks, W. Chojnacki, and R. Kozera. Shading without shape. Quarterly of Applied Mathematics, 50(1):2738, 1992.

[5] A. M. Bruckstein. On shape fron shading. Computer. Vision Graphics Image Process, 44:139-154, 1988.

[6] A.R. Bruss. The eikonal equation: Some results applicable to computer vision. Journal of Mathematical Physics, 23(5):890-896, May 1982.
[7] F. Camilli. A characterization of the value function for a class of degenerate control problems, chapter 3, pages 4758. Volume 59 of Falcone and Makridakis [21], 2001.

[8] F. Camilli and M. Falcone. An approximation scheme for the maximal solution of the shape-from-shading model. International Conference on Image Processing, pages 49 $52,1996$.

[9] F. Camilli and A. Siconolfi. Maximal subsolutions for a class of degenerate Hamilton-Jacobi problems. Indiana Univ. Math. J., 48(3):1111-1132, 1999.

[10] F. Camilli and A. Siconolfi. Hamilton-jacobi equations with measurable dependence on the state variable. $A d v$. Differential Equations, 8(6):733-768, June 2003.

[11] F. Courteille, A. Crouzil, J.-D. Durou, and P. Gurdjos. Shape from Shading en conditions réalistes d'acquisition photographique. In Proceedings of RFIA'04, 2004.

[12] M.G. Crandall. Viscosity solutions of Hamilton-Jacobi equations. In Nonlinear Problems: Present and Future, Proc. 1st Los Alamos Conf., 1981, volume 61, pages 117125. North-Holland Math. Stud., 1982.

[13] M.G. Crandall, H. Ishii, and P.-L. Lions. User's guide to viscosity solutions of second order partial differential equations. Bull. Amer. Soc., 27:1-67, 1992.

[14] M.G. Crandall and P.-L. Lions. Viscosity solutions of Hamilton-Jacobi equations. Trans. AMS, 277:1-43, 1983.

[15] P.-E. Danielsson. Euclidean Distance Mapping. Computer Graphics and Image Processing, 14(3):227- 248, November 1980 .

[16] P. Dupuis and J. Oliensis. Direct method for reconstructing shape from shading. SPIE, 1570:116-128, 1991.

[17] P. Dupuis and J. Oliensis. An optimal control formulation and related numerical methods for a problem in shape reconstruction. The Annals of Applied Probability, 4(2):287346, 1994.

[18] J-D. Durou, M. Falcone, and M. Sagona. A survey of numerical methods for shape from shading. Research report 2004-2-R, IRIT, January 2004.

[19] J.-D. Durou and H. Maître. On convergence in the methods of Strat and Smith for shape from shading. The International Journal of Computer Vision, 17(3):273-289, 1996.

[20] J.-D. Durou and D. Piau. Ambiguous shape from shading with critical points. Journal of Mathematical Imaging and Vision, 12(2):99-108, 2000.

[21] M. Falcone and C. Makridakis, editors. numerical methods for viscosity solutions and applications, volume 59 of Series on advances in mathematics for applied sciences. World Scientific, 2001. 
[22] M. Falcone and M. Sagona. An algorithm for the global solution of the shape-from-shading model. International Conference on Image Analysis and Processing, 1:596-603, 1997. LNCS 1310.

[23] M. Falcone, M. Sagona, and A. Seghini. A scheme for the shape-from-shading model with "black shadows". In Proceedings of ENUMATH 2001, 2001.

[24] J.K. Hasegawa and C.L. Tozzi. Shape from shading with perspective projection and camera calibration. Computers and Graphics, 20(3):351-364, May 1996.

[25] B.K. Horn and M.J. Brooks, editors. Shape from Shading. The MIT Press, 1989.

[26] B.K.P. Horn. Obtaining shape from shading information. In P.H. Winston, editor, The Psychology of Computer Vision. McGraw-Hill, New York, 1975.

[27] B.K.P Horn, R.S. Szeliski, and A.L. Yuille. Impossible shaded images. IEEE Transactions on Pattern Analysis and Machine Intelligence, 15(2):166-170, 1993.

[28] A. Ishii. A simple, direct proof of uniqueness for solutions of the hamilton-jacobi equations of eikonal type. Proceedings of the American Mathematical Society, pages 247251, 1987.

[29] H. Ishii. Hamilton-Jacobi equations with discontinuous hamiltonians on arbitrary open subsets. Bull. Fac. Sci. Engrg. Chuo Univ., 28:33-77, 1985.

[30] H. Ishii. a boundary value problem of the dirichlet type for hamilton-jacobi equations. Ann. Sci. Ecole Norm. Sup., 4(16):105-135, 1989.

[31] H. Ishii and M. Ramaswamy. Uniqueness results for a class of Hamilton-Jacobi equations with singular coefficients. Comm. Par. Diff. Eq., 20:2187-2213, 1995.

[32] J. Kain and D.N. Ostrov. Numerical shape-from-shading for discontinuous photographic images. The International Journal of Computer Vision, 44(3):163-173, 2001.

[33] B. Kerautret. A robust discrete approach for shape from shading and photometric stereo. In Proceeding of the International Conference on Computer Vision and Graphics (ICCVG 2004), September 22-24, 2004 Warsaw, Poland., 2004.

[34] R. Kimmel and A.M. Bruckstein. "Global shape-fromshading”. CVGIP: Image Understanding, pages 360-369, 1995.

[35] R. Kimmel and A.M. Bruckstein. Tracking level sets by level sets : A method for solving the shape from shading problem. Computer Vision and Image Understanding, 62(2):47-58, July 1995.
[36] R. Kimmel and J.A. Sethian. Optimal algorithm for shape from shading and path planning. Journal of Mathematical Imaging and Vision, 14(2):237-244, May 2001.

[37] R. Klette, R. Kozera, and K. Schlüns. Shape from shading and photometric stereo methods. Technical Report CITRTR-20, CITR, University of Auckland, New Zealand, 1998.

[38] R. Kozera. Uniqueness in shape from shading revisited. International Journal of Mathematical Imaging and Vision, 7:123-138, 1997.

[39] R. Kozera. An overview of the shape from shading problem. Machine Graphics and Vision, 7(1-2):291-312, 1998.

[40] K.M. Lee and C.C.J. Kuo. Shape from shading with perspective projection. CVGIP: Image Understanding, 59(2):202-212, 1994.

[41] P.-L. Lions. Generalized Solutions of Hamilton-Jacobi Equations. Number 69 in Research Notes in Mathematics. Pitman Advanced Publishing Program, 1982.

[42] P.-L. Lions, E. Rouy, and A. Tourin. Shape-from-shading, viscosity solutions and edges. Numer. Math., 64:323-353, 1993.

[43] T. Okatani and K. Deguchi. Reconstructing Shape from Shading with a Point Light Source at the Projection Center: Shape Reconstruction from an Endoscope Image. In Proceedings of ICPR'96, pages 830-834, August 1996.

[44] T. Okatani and K. Deguchi. Shape reconstruction from an endoscope image by shape from shading technique for a point light source at the projection center. Computer Vision and Image Understanding, 66(2):119-131, May 1997.

[45] J. Oliensis. Shape from shading as a partially wellconstrained problem. CVGIP: Image Understanding, 54(2):163-183, 1991.

[46] J. Oliensis and P. Dupuis. A global algorithm for shape from shading. In Proceedings of ICCV'93, pages 692-701, 1993.

[47] D.N. Ostrov. Extending viscosity solutions to eikonal equations with discontinuous spatial dependence. Nonlinear Anal., 42(4):709-736, 2000.

[48] M.A. Penna. Local and semi-local shape from shading for a single perspective image of a smooth object. Computer Vision, Graphics, and Image Processing, 46(3):346-366, 1989.

[49] M.A. Penna. A shape from shading analysis for a single perspective image of a polyhedron. IEEE Transactions on Pattern Analysis and Machine Intelligence, 11(6):545554, June 1989. 
[50] E. Prados and O. Faugeras. Une approche du "Shape from Shading" par solutions de viscosité. Master's thesis, University of Nice Sophia-Antipolis, France, INRIA, September 2001 .

[51] E. Prados and O. Faugeras. A mathematical and algorithmic study of the lambertian SFS problem for orthographic and pinhole cameras. Technical Report RR-5005, INRIA, November 2003.

[52] E. Prados and O. Faugeras. "Perspective Shape from Shading" and viscosity solutions. In Proceedings of the 9th International Conference on Computer Vision, volume 2, pages 826-831, October 2003.

[53] E. Prados, O. Faugeras, and E. Rouy. Shape from shading and viscosity solutions. In A. Heyden, G. Sparr, M. Nielsen, and P. Johansen, editors, Proceedings of the 7th European Conference on Computer Vision, volume 2351, pages 790-804, Copenhagen, Denmark, May 2002. Springer-Verlag.

[54] E. Prados, O. Faugeras, and E. Rouy. Shape from shading and viscosity solutions. Technical Report 4638, INRIA, November 2002.

[55] E. Rouy and A. Tourin. A Viscosity Solutions Approach to Shape-from-Shading. SIAM Journal of Numerical Analysis, 29(3):867-884, June 1992.

[56] D. Samaras and D.N. Metaxas. Coupled lighting direction and shape estimation from single images. In Proceedings of the 7th International Conference on Computer Vision, volume 2, pages 868-874, Kerkyra, Greece, September 1999. IEEE Computer Society, IEEE.

[57] J.A. Sethian. Level Set Methods. Cambridge University Press, 1996.

[58] P. Soravia. Optimal control with discontinuous running cost: eikonal equation and shape from shading. In 39th IEEE Conference on Decision and Control, pages 79-84, December 2000.

[59] A. Tankus, N. Sochen, and Y. Yeshurun. A new perspective [on] Shape-from-Shading. In Proceedings of the 9th International Conference on Computer Vision, volume 2, pages 862-869, October 2003.

[60] A. Tankus, N. Sochen, and Y. Yeshurun. Perspective Shape-from-Shading by Fast Marching. In IEEE Conference on Computer Vision and Pattern Recognition, Washington, DC (United States), June 2004.

[61] A. Tankus, N. Sochen, and Y. Yeshurun. Shape-fromshading under perspective projection. International Journal of Computer Vision, 63(1):21-43, june 2005.
[62] I. Weiss. A perspective 3D formalism for shape from shading. In Proceedings of DARPA Image Understanding Workshop, volume 2, pages 1393-1402, May 1997.

[63] S.Y. Yuen, Y.Y. Tsui, Y.W. Leung, and R.M.M. Chen. Fast marching method for shape from shading under perspective projection. In Proceedings of VIIP'02, pages 584-589, September 2002.

[64] R. Zhang, P.-S. Tsai, J.-E. Cryer, and M. Shah. Shape from Shading: A survey. IEEE Transactions on Pattern Analysis and Machine Intelligence, 21(8):690-706, August 1999. 